\title{
Reduced Leakage Synchrophasor Estimation: Hilbert Transform plus Interpolated DFT
}

\author{
Guglielmo Frigo, Asja Derviškadić, and Mario Paolone
}

\begin{abstract}
Synchrophasor estimation is typically performed by means of spectral analysis based on the Discrete Fourier Transform (DFT). Traditional DFT approaches, though, suffer from several uncertainty contributions due to the stationarity assumption, spectral leakage effects and the finite-grid resolution. The present paper addresses these limitations, by proposing a joint application of the Hilbert Transform (HT) and the interpolated DFT (IpDFT) technique. Specifically, the HT enables the suppression of the spectral leakage generated by the negative image of the tones under analysis, whereas the IpDFT limits the effects of spectrum granularity. In order to relax the constraint in terms of measurement reporting latency, the proposed estimator can adopt a window length of $40 \mathrm{~ms}$ and yet provides a noticeable estimation accuracy with a worstcase Total Vector Error and Frequency Error equal to 0.02\% and $4 \mathrm{mHz}$, respectively, in steady-state conditions. In this context, the paper discusses the most suitable setting of the algorithm parameters and their effect on spurious component rejection. Moreover, a thorough metrological characterization of the algorithm estimation accuracy and responsiveness with respect to the IEEE Std. C37.118.1 is carried out in order to detect the main uncertainty sources as well as possible room for enhancement. Finally, a comparison with two consolidated IpDFT approaches shows the actual performance enhancement provided by the proposed algorithm.
\end{abstract}

Index Terms-Phasor Measurement Unit (PMU), synchrophasor, Hilbert Transform, interpolated DFT, IEEE Std. C37.118.1.

\section{INTRODUCTION}

Monitoring and automation of power grids are growing in importance and the recent literature has discussed the application of Phasor Measurement Units (PMUs) to provide measurements of voltage and current phasors synchronized to Universal Time Coordinates (UTC) [1], [2], as they guarantee a promising trade-off between measurement accuracy and latency [3], [4]. A generic PMU is expected to provide an estimate of the fundamental amplitude, phase, frequency and Rate Of Change Of Frequency (ROCOF) with a reporting rate in the order of some tens of frames per second. In this regard, the IEEE Std. C37.118.1 [5] and its recent amendment [6], briefly IEEE Std, introduce two different performance classes: class $\mathrm{M}$ is intended for monitoring applications where high accuracy levels are required, whereas class $\mathrm{P}$ is conceived for protection applications where high responsiveness is recommended. As regards the performance characterization, the estimation accuracy is quantified in terms of Total Vector Error (TVE), Frequency Error (FE) and ROCOF Error (RFE), whose limits vary as function of the considered test conditions. The IEEE Std also introduces a maximum measurement reporting latency, defined as the maximum time interval between the measurement time-stamp, and the time when the measurement becomes available at the PMU output.

The stringent requirements in terms of accuracy have pushed the development of highly-sophisticated algorithms for synchrophasor estimation (SE), capable of rejecting spurious contributions as well as accounting for fundamental dynamics [7], [8]. In view of a PMU practical application, though, also latency limits have to be taken into account [9]. In particular, the IEEE Std requires the maximum latency not to exceed two or five times the reporting period for class $\mathrm{P}$ and $\mathrm{M}$, respectively, i.e. not to exceed some tens of milliseconds. To this end, any possible delay contribution has to be minimized, starting from the computational complexity of the adopted SE algorithm. In literature, most of the existing approaches address synchrophasor estimation as a spectral analysis problem [10]. Given a finite series of samples (i.e. a signal window), the application of the Discrete Fourier Transform (DFT) enables us to retrieve the information associated to the fundamental component. However, traditional DFT approaches suffer from several uncertainty contributions, due to the stationarity assumption, the finite-grid resolution, and spectral leakage effects [11]. In order to solve these limitations, the recent literature has proposed several solutions, ranging from interpolation techniques [8], [12], Taylor-series expansions [13]-[16], and super-resolution approaches [17]. Nevertheless, the longer the window length and the higher the computational complexity, the higher the group delay introduced by the synchrophasor estimation process. Most of current PMUs attempt to compensate for the increased group delay by shifting the measurement time-stamps. However, this solution produces a higher reporting latency that risks to affect the performance of mission-critical PMU applications [18].

Typically, DFT-based algorithms adopt a minimum window length equal to $60 \mathrm{~ms}$, corresponding to three cycles at the nominal system frequency $(50 \mathrm{~Hz})$. In fact, a shorter window might result in a too scarce frequency resolution and an intractable distortion level. In this regard, the main contribution to estimation uncertainty is represented by the interference due to the image of the fundamental component at negative frequency. It should be noticed that this phenomenon, ascribable to long-range spectral leakage, is inherent in the definition of DFT of a real-valued signal [19].

In this context, the Hilbert Transform (HT) might represent a promising and effective solution. Given the acquired waveform, in ideal conditions the HT produces a complexvalued signal, also known as the analytic signal, whose spectrum consists only of positive frequency components. 
In practical applications, we can largely mitigate the effects of long-range spectral leakage that calls for dedicated and complex algorithms [8], [12], [13], [20], and provide accurate synchrophasor estimates even on reduced window lengths, up to just two cycles at nominal system frequency, i.e. $40 \mathrm{~ms}$ (corresponding to a frequency resolution of $25 \mathrm{~Hz}$ ).

In more detail, the proposed algorithm consists of three consecutive steps. First, we compute the analytic signal associated to the acquired waveform by means of the HT. Then, we apply a Hanning weighing function. Finally, we adopt a three-point interpolation technique to estimate the fundamental amplitude, frequency and initial phase. For this reason, we identify the algorithm as HT-IpDFT, that accounts for both leakage suppression and finite resolution compensation.

It is worth noticing that the HT has been previously applied to the analysis of power signals, because of the capability to extract an instantaneous envelope of the signal under analysis [21]. This peculiar property is typically employed, in combination with singular value decomposition [22] or wavelet transform [23], for a nearly real-time monitoring of power quality indices. In [24], HT is applied to the autocorrelation of the acquired power signal to design an innovative P-class PMU for protection applications.

The paper is organized as follows. In Section II we introduce the mathematical formulation of the HT, and discuss its possible implementations. Section III describes the main stages of the proposed HT-IpDFT algorithm and defines the criteria for setting the parameters. In Section IV we thoroughly characterize the estimation accuracy in all the static and dynamic test conditions indicated by the IEEE Std with specific reference to the compliance requirements for both class $\mathrm{P}$ and $\mathrm{M}$. Section V compares the proposed algorithm with two other IpDFT-based solutions, that attempt to mitigate long-range spectral leakage. Finally, in Section VI we provide some closing remarks.

\section{HilbERT TRANSFORM}

We consider a time-variant noise-less power signal affected by disturbances, modeled as follows:

$$
x(t)=A\left(1+\varepsilon_{A}(t)\right) \cdot \cos \left(2 \pi f t+\varphi_{0}+\varepsilon_{\varphi}(t)\right)+\eta(t)
$$

where $A, f$ and $\varphi_{0}$ are the amplitude, frequency and initial phase of the fundamental component, respectively ${ }^{1}$. The timevarying terms $\varepsilon_{A}(t)$ and $\varepsilon_{\varphi}(t)$ represent amplitude and phase fluctuations, whereas $\eta(t)$ accounts for possible sources of spurious contributions, e.g. DC, harmonic or inter-harmonic components and, in general, any transient event. It is worth observing that, based on (1), it is possible to reproduce any test condition provided by the IEEE Std (see Section IV).

In the context of PMU-based applications, we typically acquire a discrete time-series of samples, defined as follows:

$$
x[n]:=\left\{x(t)+\rho(t) \quad \mid \quad t=n T_{s}, n=\left[1, \ldots N_{m}\right] \in \mathbb{N}\right\}
$$

\footnotetext{
${ }^{1}$ Unless otherwise indicated, we consider a power system scenario within the synchronous grid of Continental Europe, where the nominal values of $A$, $f$ and $\varphi_{0}$ are equal to $1 \mathrm{pu}, 50 \mathrm{~Hz}$ and $0 \mathrm{rad}$, respectively.
}

where $N_{m}$ is the number of samples that compose the considered window $x[n]$, and $\rho(t)$ accounts for wide-band disturbances (e.g additive uncorrelated noise). For the sake of simplicity, we assume a uniform sampling rate $F_{s}=T_{s}^{-1}$.

Given $x[n]$, synchrophasor estimation algorithms attempt to retrieve the instantaneous value of $A, \varphi_{0}, f$, and ROCOF with a given reporting rate $F_{r}$.

The HT $\mathcal{H}(\cdot)$ is defined as a convolution operator with the function $1 / \pi t$ :

$$
\tilde{x}(t)=\mathcal{H}[x(t)]=\frac{1}{\pi} \int_{-\infty}^{+\infty} \frac{x(\tau)}{t-\tau} d \tau
$$

It should be noticed that the integrand function presents a singularity in $\tau=t$, thus (3) does not yield a closed-form solution. In this regard, an approximation can be obtained by applying the Cauchy Principal Value method as follows [25]:

$$
\mathcal{H}[x(t)] \simeq \frac{1}{\pi} \lim _{\varepsilon \rightarrow 0^{+}}\left[\int_{-\infty}^{t-\varepsilon} \frac{x(\tau)}{t-\tau} d \tau+\int_{t+\varepsilon}^{+\infty} \frac{x(\tau)}{t-\tau} d \tau\right]
$$

In this way, it is possible to define the HT of some basic and frequently employed functions [26], e.g. $\mathcal{H}[\cos (t)]=\sin (t)$.

In addition to the convolution operator formulation, the HT can be also interpreted as a linear phase-shift filter, characterized by a frequency response $H(\cdot)$ defined as follows:

$$
H(\omega)=-j \cdot \operatorname{sgn}(\omega)
$$

where $\omega$ and $j$ represent the considered angular frequency and the imaginary unit, respectively. Accordingly, the HT filter exhibits a Hermitian symmetric trend with respect to the DC frequency, i.e. it keeps unaltered the spectral component magnitudes, while producing a phase shift equal to $-\operatorname{sgn}(\omega)$. $\pi / 2$.

In this context, let us introduce the analytic signal $\hat{x}(t)$ :

$$
\hat{x}(t)=x(t)+j \cdot \mathcal{H}[x(t)]=x(t)+j \cdot \tilde{x}(t)
$$

defined as a complex-valued quantity, with the original signal and the HT filter output as real and imaginary component, respectively. From (5) and (6), we observe that the spectral representation of the analytic signal does not consist of negative frequency components, but only of (doubled) positive frequency components. It is thus reasonable to expect that the DFT of the analytic signal is less affected by long-range spectral leakage effects.

It should be noticed that the removal of the negative frequency components does not correspond to a loss or alteration of the original information content. From a mathematical point of view, the analytic signal can be considered as a generalization of the concept of phasor representation. Given a phasor model, we are able to provide an instantaneous estimate of the signal amplitude, frequency and phase, under the assumption that such quantities are timeinvariant. The analytic signal, instead, allows for tracking the time-variations of the signal parameters [27].

In this context, Fig. 1(a) compares the DFT representations of three different signals: a real-valued cosine signal $x(t)$, the corresponding analytic signal $\hat{x}(t)$, and a DFT pure tone $w(t)$ 


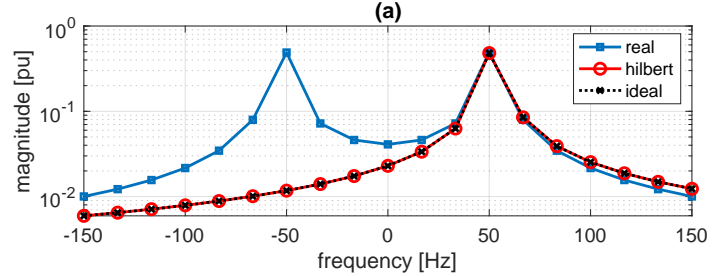

(b)

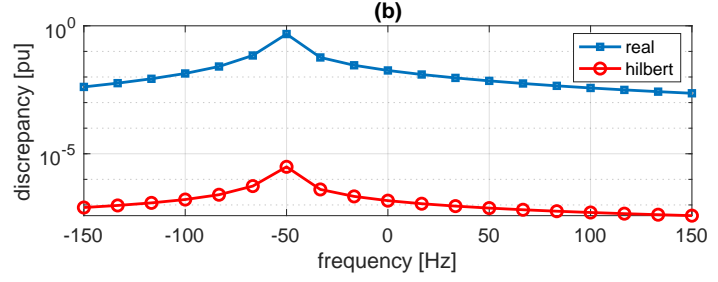

Fig. 1. In (a), DFT of a real-valued cosine signal (blue, squares), the analytic signal obtained by its HT (red, circles), and the ideal spectral representation (black, crosses). In (b), discrepancy between real-valued and analytic signal with respect to ideal spectral representation (only positive frequency).

(usually referred to as DFT kernel) with the same amplitude, frequency and initial phase:

$$
\begin{aligned}
x(t) & =A \cdot \cos \left(2 \pi f t+\varphi_{0}\right) \\
\hat{x}(t) & =A / 2 \cdot\left(\cos \left(2 \pi f t+\varphi_{0}\right)+j \cdot \sin \left(2 \pi f t+\varphi_{0}\right)\right) \\
w(t) & =A / 2 \cdot \exp ^{j \cdot\left(2 \pi f t+\varphi_{0}\right)}
\end{aligned}
$$

More precisely, the signal under investigation is characterized by $A, f$ and $\varphi_{0}$ equal to $1 \mathrm{pu}, 52.5 \mathrm{~Hz}$ and $0 \mathrm{rad}$, respectively, while the sampling rate is $50 \mathrm{kHz}$. In this case, the window length consists of three nominal cycles, i.e. 60 $\mathrm{ms}$, corresponding to a frequency resolution of $16.67 \mathrm{~Hz}$. This window length has been selected in order to enhance the graphical representation of spectral leakage effects, but similar results hold for any window length. For the sake of a clearer comparison, both the analytic and pure tone signals have been halved, therefore the DFT peak values coincide.

Due to incoherent sampling, all the spectral representations are affected by spectral leakage. Nevertheless, it is interesting to observe how the analytic signal presents only the lobe associated to the positive frequency component. In this regard, Fig. 1(b) presents the point-wise deviation of real-valued and analytic signal DFTs with respect to the complex exponential reference. It should be noticed that the suppression of the negative frequency component is nearly perfect: the remainder spurious contribution is in the order of some parts per million, probably due to the limited spectral resolution given by the finite number of samples employed in this example. Based on this property, it is reasonable to expect that DFT-based algorithms might provide accurate estimates also on restricted window lengths, i.e shorter than $60 \mathrm{~ms}$.

As aforementioned, though, the HT computation represents an open issue. Indeed, we cannot know in advance the exact signal model, thus an approximation of the HT should rely exclusively on the acquired time-series $x[n]$. In this context, two are the possible solutions. On one side, the frequencybased methods apply a weighing function to the signal DFT, to approximate (5), and retrieve the analytic signal through the inverse DFT [28], [29]. Such methods can be easily implemented, but are affected by the typical DFT limitations, e.g. stationarity constraint and truncation effects. In this regard, it is worth observing that the DFT relies on a static signal model, that does not comply with time-varying test conditions [30]. For this reason, in the presence of dynamic trends, it is reasonable to expect that the signal DFT accounts only for an average representation of the parameter variations. On the other side, the time-based methods approximate (5) by means of a cascade of Infinite Impulse Response (IIR) filters [31]. These methods can operate in nearly real-time conditions, even if that requires an accurate characterization and compensation of filter contributions in terms of magnitude gain and phase delay. Moreover, the employment of digital filters does not require the computation of both direct and inverse DFT, and better preserves the original signal dynamics [32]. For this reason, in the present paper we propose a time-based method that employs two different filter banks, whose implementation details are provided in the next section.

\section{Algorithm Description}

In this section, we describe the main steps of the proposed HT-IpDFT algorithm and discuss the theoretical and experimental criteria that condition the choice of parameter values.

The block scheme in Fig. 2 identifies the algorithm dataflow, organized in two sequential stages committed to filtering and estimation operations, respectively.

In the first stage, the acquired signal passes through a bank of two parallel HT filters, characterized by high reconstruction accuracy and responsiveness, respectively. An Event Detection Block determines which filter output has to be propagated to the following stage. If a transient event is detected, the best option is represented by the Fast HT Filter, capable

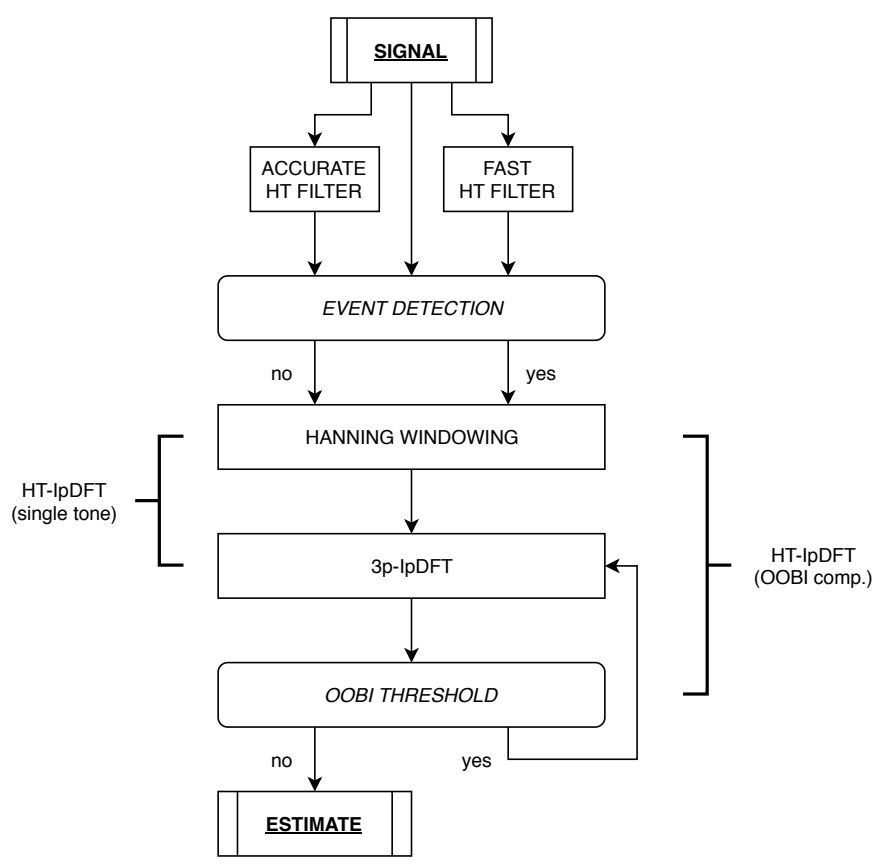

Fig. 2. Block scheme of the proposed HT-IpDFT algorithm. 
of promptly responding to rapid variations. Otherwise, the Accurate HT Filter is preferable, because of its reduced distortion effect.

In the second stage, we apply a Hanning Window to the selected HT filter output and employ a Three-Point Interpolated DFT (3p-IpDFT) algorithm to estimate the amplitude, frequency and initial phase of the fundamental component. Based on this preliminary estimate, we subtract the fundamental component contribution and we verify whether the resulting spectrum still contains spurious narrowband components. To this end, we apply an Energy Thresholding Process to the DFT bins in a restricted bandwidth, i.e. $[0,150] \mathrm{Hz}$. If the energy content is larger than the expected noise level, an iterative compensation routine is triggered. Otherwise, the obtained estimates are validated as the final HT-IpDFT outcomes.

In the following paragraphs, we will briefly describe the implementation details of each stage and define the criteria for the parameters' setting.

a) Filtering stage: In order to meet the IEEE Std stringent requirements in terms of accuracy and responsiveness, we design and implement in Matlab R2018a two different filters that approximate the HT properties. Both rely on a two-stage cascade of IIR filters, whose parameters and performance indices are reported in Table I where a sampling frequency of $50 \mathrm{kHz}$ is assumed. It should be noticed that the IIR implementation allows to approximate the ideal HT frequency response with a sufficiently restrained computational complexity. The main disadvantage of IIR filters is typically represented by their non-linear phase response. Nevertheless, the present analysis focuses on a restricted bandwidth, i.e $[10,150] \mathrm{Hz}$, where the filter response can be reasonably considered as almost linear and thus can be properly compensated. Once characterized the filter response as function of frequency, it is possible to determine the amplitude and phase correction based on the estimated value of the fundamental frequency.

In this context, it should be noticed that the solution proposed in the present paper allows for an effective mitigation of long-range spectral leakage effects. Nevertheless, the PMU developer can choose among many other implementation alternatives based on the adopted hardware platform and processing capabilities. In this context, the main objective consists only in obtaining the most suitable approximation of the frequency response given in (5).

As regards the filter design, we set the same transition width of $50 \mathrm{~Hz}$, thus guaranteeing a flat pass-band response within the range $[25,75] \mathrm{Hz}$, that corresponds to the pass-bandwidth of a PMU that assumes a nominal fundamental frequency of $50 \mathrm{~Hz}$ and a reporting rate of $50 \mathrm{fps}$. In the design process, we aim at minimizing the pass-band ripple and the power density of the round-off noise, in order not to introduce further uncertainty sources in the acquired waveforms. To this end, we design two different filters, whose order is set equal to 61 and 21 , for the accurate and fast solution, respectively.

In order to evaluate the filter performance, we consider four different aspects: the pass-band ripple, the group delay, the power spectral density of the round-off noise, and the
TABLE I

HT FILTER PARAMETERS

\begin{tabular}{c|cc}
\hline Parameter & Accurate & Fast \\
\hline filter order & 61 & 21 \\
stages number & 2 & 2 \\
transition width [Hz] & 50 & 50 \\
pass-band ripple [dB] & $8.37 \cdot 10^{-10}$ & $8.86 \cdot 10^{-6}$ \\
group delay [ms] at 50 Hz & 4.6 & 5.7 \\
round-off noise [dB/Hz] at 50 Hz & -89.3 & -99.8 \\
multipliers number & 32 & 12 \\
adders number & 61 & 21 \\
states number & 65 & 25 \\
\hline
\end{tabular}

computational complexity. The pass-band ripple accounts for the deviation from unity gain in the filter pass-bandwidth. In this regard, the accurate solution is characterized by a ripple lower than $8.5 \cdot 10^{-10} \mathrm{~dB}$, whereas the fast solution provides a ripple in the order of $8.9 \cdot 10^{-6} \mathrm{~dB}$. The group delay, instead, quantifies the time delay between the input and output of the filter cascade. Since IIR filters are characterized by group delays varying with the frequency of the test signal, in Table I we report the value related to the nominal fundamental frequency, $50 \mathrm{~Hz}$. In particular, we measure a group delay of 4.6 and $5.7 \mathrm{~ms}$ for accurate and fast solution, respectively.

The round-off noise is produced by the quantization and truncation error within the filter cascade, due to the finite precision arithmetic. In line with the group delay analysis, we focus on the noise power within the bandwidth of interest. In particular, at $50 \mathrm{~Hz}$ the accurate solution is characterized by a noise power spectral density in the order of $-90 \mathrm{~dB} / \mathrm{Hz}$. Thanks to the reduced number of coefficients, the fast solution guarantees an even lower noise power, around $-100 \mathrm{~dB} / \mathrm{Hz}$. Finally, we evaluate the computational complexity of the designed filters. In particular, we determine the number of multipliers, adders and filter internal states, necessary to implement the filter cascades. In this context, the accurate solution proves to be more demanding than the fast solution. In fact, the more precise approximation of the peculiar HT frequency response is achieved at the price of higher number of states and operations.

Fig. 3(a) represents the magnitude and phase response of the Accurate HT Filter over the entire acquisition bandwidth. It is worth observing that the magnitude response (blue line) is nearly equal to $0 \mathrm{~dB}$ for the positive frequency components, whereas it decreases from -60 up to $-140 \mathrm{~dB}$ in the negative frequency domain. In accordance with (5), the phase response (red line) produces a shift of $\pi / 2$ for the negative frequency components and $-\pi / 2$ for the positive frequency ones. In this way, it is reasonable to expect that a significant rejection of the long-range interference can be achieved.

A detailed view of the trend over the PMU pass-bandwidth $[25,75] \mathrm{Hz}$ is provided in Fig. 3(b). Both magnitude and phase response are not equal to their ideal values, i.e. $0 \mathrm{~dB}$ and $-\pi / 2$, respectively. Nevertheless they exhibit a nearly linear trend that can be easily compensated, once the frequency of the fundamental component has been estimated. Similar trends can be obtained on varying the filter order (e.g. from 61 to 21) or the sampling rate (e.g. from 50 to $10 \mathrm{kHz}$ ). Once designed 
the filter frequency response, we model the magnitude gain and phase delay within the range of interest $[10,150] \mathrm{Hz}$ by means of a smoothing spline approximation. In this regard, the goodness of fit is guaranteed by the fact that the models present a coefficient of determination $r^{2}$ equal to 1 , and the square root of the variance of the residuals is equal to $2.31 \cdot 10^{-4}$ and $8.82 \cdot 10^{-6}$ for the accurate and fast solution, respectively.

The selection between Accurate and Fast HT Filter outputs is carried out by an Event Detection Block that relies on a simple feedback operation. First, we process the input signal through a Butterworth third-order low-pass filter. We set the cut-off frequency equal to $3 \mathrm{kHz}$, in order to include within the filter pass-bandwidth any harmonic term up to $50^{\text {th }}$ order. As shown in Fig. 4(a), the stop-band is characterized by an attenuation of $-20 \mathrm{~dB}$. Then, we compute the discrepancy, i.e. the point-wise difference, between the original and filtered version of the input signal: if it exceeds the expected level of variability (due to measurement noise and filter distortion) a transient event is detected.

In order to define the threshold level, we carry out a heuristic procedure and we evaluate the distribution of the discrepancy values in the test conditions indicated by the IEEE Std with a test duration of $5 \mathrm{~s}$, a sampling frequency of $10 \mathrm{kHz}$, and a signal-to-noise ratio of $60 \mathrm{~dB}$. In this regard, it should be noticed that we consider the most challenging among the considered scenarios, as it is characterized by the highest noise level and the poorest time resolution. As it can be seen in Fig. 4(b), in the presence of amplitude and phase steps (AS and PS, respectively) the discrepancy presents some outliers that can be used to detect the occurrence of a step change with a delay of less than 10 samples. In the presence of abrupt variations (e.g., an amplitude step change), the filter output presents a slower transition, due to the finite impulse response and the limited pass-bandwidth. As a consequence, the discrepancy value is expected to exceed its typical variability depending on the test signal characteristics, the SNR and the sampling frequency. Based on this analysis, the event detection threshold (black dashed line) is set equal to $17.5 \cdot 10^{-3} \mathrm{pu}$, larger than the maximum discrepancy obtained during high-order harmonic
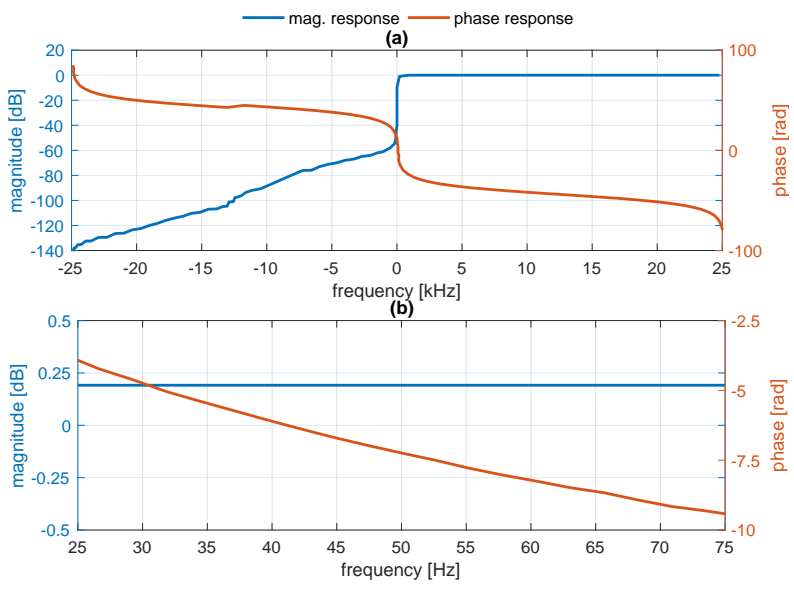

Fig. 3. Frequency response of the Accurate HT Filter for a sampling rate of $50 \mathrm{kHz}$, over the acquisition (a) and the PMU pass-bandwidth (b). distortion (HD-2500), but lower than the outliers produced by amplitude and phase step. Due to its non-linear dependence by the test conditions, the event detection process might be improperly triggered in the presence of high-order harmonic tones. Nevertheless, the presence of a single harmonic tone whose amplitude and frequency are equal to $0.1 \mathrm{pu}$ and $2500 \mathrm{~Hz}$ is rather unrealistic in practice, as it would imply a carryover of $10 \%$ of the signal energy at high frequency. Furthermore, it is worth noticing that this kind of interference does not produce significant distortion effects on the DFT bins interested by the interpolation process. Therefore, even in case of erroneous detection, also the Fast HT filter guarantees a sufficient level of estimation accuracy (as shown by TVE and FE results in Sec. IV.c).

b) Estimation stage: Once computed the analytic signal $\hat{x}(t)$, we estimate the fundamental amplitude, frequency and initial phase through an approach based on the IpDFT.

This technique enables us to mitigate the detrimental effects of spectral finite resolution by interpolating the highest amplitude DFT bins of the signal spectrum [19], as well as to reduce the effects of spectral leakage thanks to the application of bell-shaped windowing functions [11].

In the present case, we adopt the following configuration:

- Hanning window function, for the good trade-off between the main-lobe width and side-lobe levels [33];

- three-point interpolation, as the multiple-point schemes inherently reduce long-term spectral leakage effects [34].

By considering the analytic signal $\hat{x}(t)$, we would expect to deal with spectra containing the positive frequency components only. In the absence of spurious interfering components, the long-term spectral leakage should be almost negligible. However, since the HT filter is only an approximation of the ideal transform, residual distortion contribution might still be present, and should be suitably
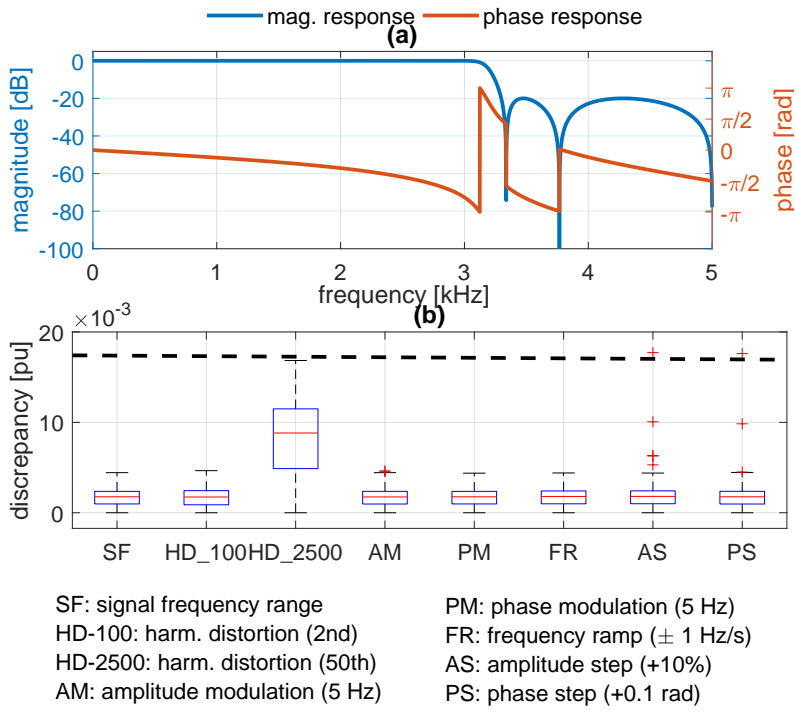

Fig. 4. Event Detection Block: magnitude and phase response (wrapped to the range $[-\pi,+\pi]$ ) of the low-pass filter (a), boxplot representation of the discrepancy value distributions in different IEEE Std tests with respect to selected threshold level (b). 
mitigated, in particular when a two-cycle window length is employed.

As any other IpDFT-based approach, also the HT-IpDFT is affected by two accuracy issues. On one side, it relies on a static signal model, i.e. amplitude, frequency and phase are assumed to be time-invariant within the considered observation window. On the other side, the interpolation technique does not account for distortion contributions coming from spurious components (either harmonic or inter-harmonic).

As regards the static signal model, it is worth pointing out that this assumption does not hold in practical operating conditions, when several dynamic trends might affect the signal parameters. In this context, it is necessary to make specific distinctions. If a transient event (e.g. a step change of amplitude or phase) occurs, the signal energy content is spread over the entire spectrum and cannot be analyzed by canonical spectral analysis methods characterized by finite frequency resolution, as further discussed in Sec. IV.c. Instead, if the signal is affected by a modulation or linear variation, the observed dynamic is typically limited (e.g. the IEEE Std considers a maximum modulation frequency of $5 \mathrm{~Hz}$ ). In this sense, a shorter window length allows for reducing the discrepancy between signal model and acquired waveform, and thus reducing the estimation errors.

In terms of rejection of spurious contributions, instead, the IpDFT produces inaccurate and unreliable results in presence of interfering components that are relatively close to the main one, such those defined in the IEEE Std Out of band interference (OOBI) test. To this end, an iterative routine has been developed to identify the interfering component parameters and suitably compensate their contribution in the DFT bins associated to the fundamental component [8].

First, we approximate the spectral trend of the positive frequency component associated to the estimates $A^{*}, f^{*}$ and $\varphi_{0}^{*}$, and subtract it from the original DFT bins to produce a spectrum that accounts for any remainder spurious contribution. Then, we compute the ratio between the residual and original spectrum energy $E_{r}$ and compare it with a threshold level, defined on the basis of the expected uncertainty level due to measurement noise and algorithm accuracy. If the threshold is exceeded, a peak search identifies the plausible bin associated to the interfering component and a new three-point interpolation is performed. Once identified the spurious component parameters, they are subtracted from the original DFT bins and a new fundamental estimation is performed.

In order to guarantee feasibility and efficiency, we introduce two stop criteria. More precisely, the routine stops when the incremental ratio between two consecutive values of $E_{r}$ is negligible, i.e. lower than $10^{-10}$, or when the iteration number exceeds the maximum limit $Q$ equal to 100 .

In the present case, we apply this compensation routine only to inter-harmonic and harmonic components whose frequency does not exceed $150 \mathrm{~Hz}$. In fact, higher-frequency components do not produce significant distortion effects within the DFT bins associated to the fundamental component. Therefore, the energy computation and thresholding process can be restricted to a set of bins, whose cardinality $K$ depends on the adopted
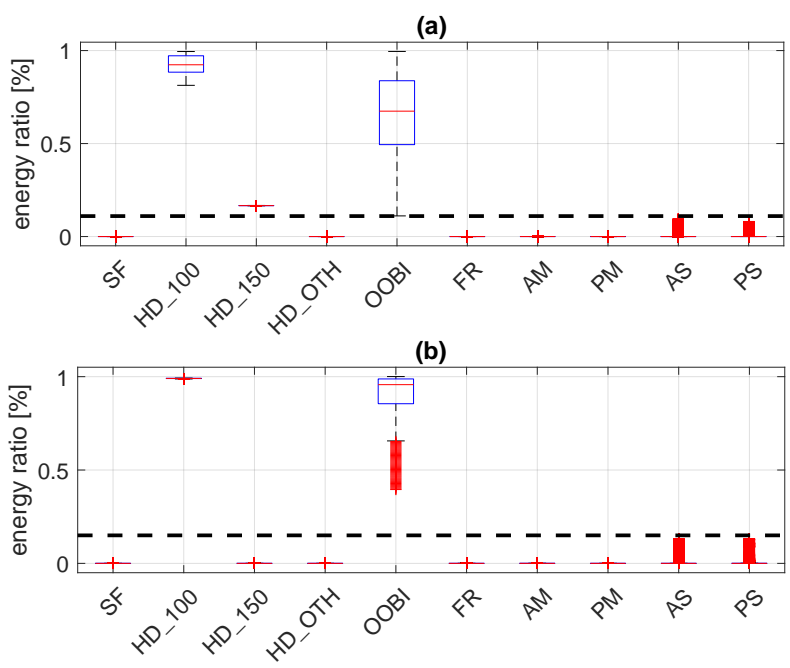

SF: signal frequency range HD-100: harm. distortion (2nd) HD-150: harm. distortion (3rd) HD-OTH: harm. dist. ( $\geq 4$ th) OOBI: out-of-band interference
FR: frequency ramp $( \pm 1 \mathrm{~Hz} / \mathrm{s})$ AM: amplitude modulation $(5 \mathrm{~Hz})$ PM: phase modulation $(5 \mathrm{~Hz})$

AS: amplitude step (+10\%)

PS: phase step $(+0.1 \mathrm{rad})$
Fig. 5. Iterative Compensation Routine: boxplot representation of the energy ratio value in different IEEE Std tests with respect to selected threshold level with window length equal to two (a) and three cycles (b).

window length, i.e. 5 and 8 bins for 40 and $60 \mathrm{~ms}$, respectively.

In order to define the most suitable detection threshold, we carry out a heuristic procedure to determine the distribution of $E_{r}$ in the test conditions indicated by the IEEE Std, with a sampling frequency of $10 \mathrm{kHz}$ and a signal-tonoise ratio (SNR) of $60 \mathrm{~dB}$. As stated before for the event detection threshold, this particular condition represents the most challenging scenario among the ones reproduced in this analysis. In this regard, Fig. 5 shows the box-plot associated to the different test conditions for window length equal to 40 and $60 \mathrm{~ms}$, respectively. For this analysis, interfering component amplitudes are set equal to $0.1 \mathrm{pu}$, i.e. the maximum value required by the IEEE Std.

It is interesting to observe that the threshold value is nearly coincident in the considered two cases $(0.11 \%$ for two-cycles, $0.15 \%$ for three-cycles) and enables us to discriminate between the presence of spurious components with frequency lower than $150 \mathrm{~Hz}$, whereas higher-order harmonics (HD-OTH) or dynamic test conditions do not trigger the iterative routine.

In both the configurations, the OOBI test is characterized by a significantly large variability, due to the wide range of frequencies interested by the interfering tones, i.e. [10, 25] $\mathrm{Hz}$ and $[75,95] \mathrm{Hz}$. As regards the third-order harmonic, it is worth noticing that it exceeds the threshold only in two-cycle configuration. In this case, the reduced frequency resolution and the Hanning windowing produce the tails of the harmonic component to interfere with the tails of the fundamental one. In the tree-cycle configuration, instead, the enhanced frequency resolution guarantees a complete separation between the two spectral contributions, as confirmed by the energy ratio distribution that coincides with the one produced by the 
higher-order harmonic components. The energy ratio might assume high values (close to the detection threshold) also in the presence of transient events, like amplitude and phase step changes. Nevertheless, in this case, the Event Detection block is triggered and the compensation routine is not activated.

c) Computational complexity: In order to assess the practicality of a future integration of HT-IpDFT into an embedded hardware solution, we evaluate the computational complexity of the proposed algorithm with respect to the Matlab implementation employed for the following simulations and characterization. In this context, Table II summarizes the number of arithmetic operations required to produce each step of the HT-IpDFT, in case the energy ratio threshold for the iterative compensation is exceeded or not.

Taking as reference a Field Programmable Gate Array (FPGA)-based device, we discriminate between simple (like additions, multiplications) and complex operations (like exponential, trigonometric operators), as well as function calls (like the call of a sub-routine or an algorithm).

In Table II, the operation count is parametrized with respect to the number of considered DFT bins K, and the iteration number $\mathrm{Q}$. The first parameter $\mathrm{K}$ depends on the window length: two- and three-cycle configurations correspond to 5 and 8, respectively. The second parameter $\mathrm{Q}$ is upper limited to 100 , but its actual value depends on the amplitude and frequency of the interfering tone. For instance, if we consider a three-cycle window, the HT-IpDFT, inclusive of the OOBI compensation routine, requires $233+223 \cdot \mathrm{Q}$ simple operations, and $32+14 \cdot \mathrm{Q}$ complex operations.

As terms of comparison, we consider the computational complexity of the e-IpDFT [12] and i-IpDFT [8] algorithms, that implement similar leakage compensation routines. In particular, the e-IpDFT algorithm applies a routine to mitigate the long-range spectral leakage due to the image of the fundamental component at the negative frequency, but is affected by a significant performance deterioration in the presence of interfering terms. Conversely, the i-IpDFT attempts to compensate also the short-range spectral leakage caused by harmonic or inter-harmonic components.

For this analysis, we consider a three-cycle window and a Hanning weighing function. In this context, the eIpDFT requires just 186 simple operations and 105 complex operations. The i-IpDFT, instead, requires 1151+2174.Q simple operations, and $713+1426 \cdot \mathrm{Q}$ complex operations, where the iteration number $\mathrm{Q}$ is upper limited to 28 . If we consider the maximum iteration number, we obtain a worstcase scenario where the number of simple operations is 23533 for HT-IpDFT and 62023 for i-IpDFT, and the number of complex operations is 1432 for HT-IpDFT and 40641 for i-IpDFT. It is thus reasonable to say that the HT-IpDFT is characterized by a noticeable estimation accuracy as well as a limited computational complexity thanks to the possibility of neglecting half of the signal spectrum (i.e. its negative frequency components).

\section{Performance Characterization}

In this section, we thoroughly characterize the estimation accuracy of the HT-IpDFT algorithm in terms of TVE and FE.
TABLE II

HT-IPDFT COMPUTATIONAL COMPLEXITY

\begin{tabular}{lccc}
\hline & & Parameter & Value \\
\cline { 2 - 4 } & & $\mathrm{Q}$ & $\leq 100$ \\
& & $\mathrm{~K}$ & $5(2 \mathrm{cyc})$. \\
& $+|-| \times$ & $:|\exp | \sin \mid<$ & funct \\
\hline & 10 & 8 & \\
\hline (A) Dirichlet kernel & 7 & 0 & $3 \times(\mathrm{A})$ \\
(B) Hanning window & 5 & 1 & $\mathrm{~K} \times(\mathrm{B})$ \\
(C) Image compensation & 20 & 6 & \\
(D) 3p-IpDFT & 21 & 6 & $(\mathrm{C})+(\mathrm{D})$ \\
(E) 3p-IpDFT (+ comp) & & 0 & $(2 \mathrm{Q}+2) \times(\mathrm{C})+$ \\
\hline HT-IpDFT (single tone) & $3 \mathrm{~K}+4$ & & $\mathrm{Q} \times(\mathrm{D}) \times(\mathrm{D})$ \\
& & 0 &
\end{tabular}

As suggested by the signal processing models in the IEEE Std Annex C, the HT-IpDFT computes ROCOF as the incremental ratio between two consecutive frequency estimates [5].

For this analysis, we reproduce in Matlab both the static and dynamic test conditions dictated by the IEEE Std. For each test condition, we compare the estimation accuracy with the most stringent and challenging requirements (independently from the performance class $\mathrm{M}$ or $\mathrm{P}$ ). In this way, we aim at proving that the HT-IpDFT is suitable for both monitoring and protection applications.

To this end, we consider four different configurations of HTIpDFT. On one side, we adopt two different window lengths, i.e. $[40,60] \mathrm{ms}$, corresponding to two and three cycles of the nominal system frequency, respectively. On the other side, we vary the sampling rate $F_{s}$ within $[10,50] \mathrm{kHz}$, that represent typical values in current commercial PMUs.

For each test condition, we reproduce a signal waveform $x[n]$ with an overall duration of $5 \mathrm{~s}$ (except for the modulation and frequency ramp tests where the duration can be increased up to $20 \mathrm{~s}$ ). In order to approximate a more realistic operating scenario, we simulate the measurement uncertainty by means of an additive white Gaussian noise component. In particular, we consider two different signal-to-noise ratios (SNRs) equal to 80 and $60 \mathrm{~dB}$, corresponding to an equivalent resolution of 13 and 10 bits, respectively.

Unless otherwise specified, the reporting rate $F_{r}$ is set equal to $50 \mathrm{fps}$, even if it can be increased up to the value of $F_{s}$ (e.g. during the interleaved analysis in Section IV.c). As a result, each test produces a time-series of TVEs, FEs and RFEs, defined according to the IEEE Std specifications as:

$$
\mathrm{TVE}=\frac{\|\hat{p}-p\|_{2}}{\|p\|_{2}} ; \quad \mathrm{FE}=\hat{f}-f ; \quad \mathrm{RFE}=\frac{\partial \hat{f}}{\partial t}-\frac{\partial f}{\partial t}
$$

where $p$ and $f$ represent the synchrophasor and frequency associated to the fundamental component, respectively, and the hat denotes the estimated values with respect to the reference true values. For each performance index, we determine the maximum error value and compare it with the corresponding requirement of class $\mathrm{M}$ and $\mathrm{P}$. 
In the following, the test results are presented in two ways. For each group of tests, a Table reports the maximum TVE and FE provided by each HT-IpDFT configuration as function of the test condition ${ }^{2}$. For the sake of conciseness, instead, the figures are limited to the configurations with $F_{s}$ and SNR equal to $50 \mathrm{kHz}$ and $80 \mathrm{~dB}$, respectively. This choice is motivated by the fact that similar values of sampling rate and resolution can be reasonably achieved by modern technology of embedded controllers and voltage and current sensors respectively.

a) Static Tests: In accordance with the IEEE Std specifications, the static tests are intended to assess the algorithm estimation accuracy in steady-state conditions. In this context, Table III reports the results of HT-IpDFT for all the static tests.

The first test, known as Signal frequency range, considers a signal model defined as follows:

$$
x(t)=A \cdot \cos \left(2 \pi f t+\varphi_{0}\right)
$$

where $A$ and $\varphi_{0}$ are fixed to their nominal values, whereas $f$ varies within $[45,55] \mathrm{Hz}$ with an incremental step of 1 $\mathrm{Hz}$. Since the fundamental frequency is not synchronized with the sampling rate, this test enables us to characterize also the estimation accuracy as function of the initial phase $\mathrm{p}^{3}$.

As reported in Table III, it is reasonable to say that in terms of TVE the main uncertainty source is represented by the additive noise rather than the window length. In the worst-case, TVE does not exceed $0.025 \%$, i.e. it is negligible if compared with the IEEE Std limit equal to $1 \%$. As regards FE, instead, it is worth observing that the window length significantly affects the estimation accuracy: passing from two to three cycles, the error is nearly halved. Nevertheless, the worst-case FE keeps strictly lower than the IEEE Std limit of $5 \mathrm{mHz}$.

Also, similar results are obtained with a sampling frequency of 10 or $50 \mathrm{kHz}$. Indeed, by increasing the sampling frequency, we are able to mitigate the effect of additive white Gaussian noise, and thus produce a significant error reduction in all the considered configurations. In the worst-case, TVE and FE do not exceed $0.015 \%$ and $2 \mathrm{mHz}$, respectively. In this regard, Fig. 6 shows how a three-cycles window guarantees more accurate frequency estimates, whilst it does not provide a comparable enhancement in terms of TVE.

In terms of RFE, the two- and three-cycle configuration provide a maximum error of 3.6 and $1.7 \mathrm{mHz} / \mathrm{s}$, respectively, largely within the limit $100 \mathrm{mHz} / \mathrm{s}$ introduced in the last amendment of the IEEE Std [6].

In the Harmonic distortion test, we use the signal model:

$$
\begin{aligned}
& x(t)=A \cdot \cos \left(2 \pi f t+\varphi_{0}\right)+\eta(t) \\
& \eta(t)=A_{h} \cdot \cos \left(2 \pi h f t+\varphi_{h}\right)
\end{aligned}
$$

where the fundamental component is characterized by nominal amplitude, frequency and initial phase, whereas the harmonic

\footnotetext{
${ }^{2}$ For the sake of brevity, the maximum RFE is not included in the tables, but is thoroughly discussed throughout the text.

${ }^{3}$ It should be noticed that the IEEE Std requires a similar characterization as function of the signal amplitude. In a simulation context, though, the vertical resolution is independent from $A$. Nevertheless, the simulation of different SNRs enables us to partially explore also this aspect.
}
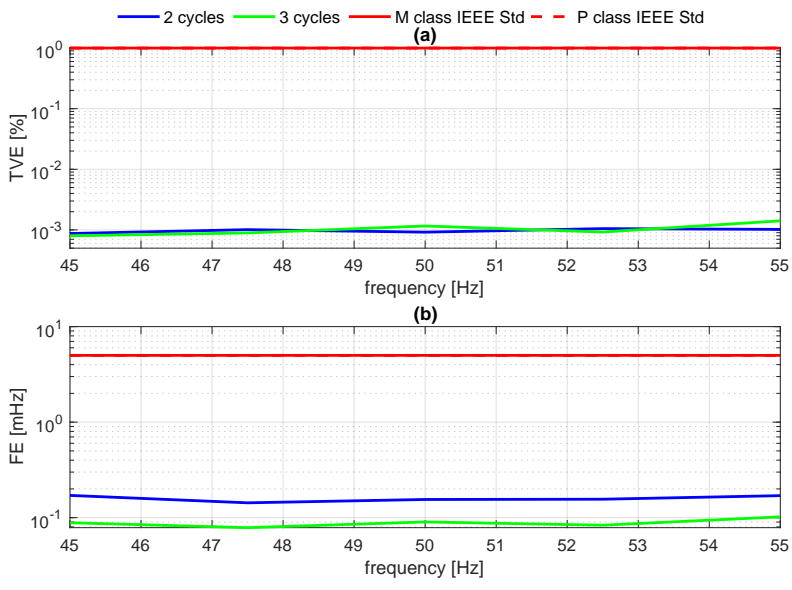

Fig. 6. Signal frequency range test: worst-case TVE (a) and FE (b) as function of signal fundamental frequency, with 2 (blue) and 3 cycles (green) window. The solid and dashed red lines represent the performance requirements for $\mathrm{M}$ - and P-class, respectively.

distortion is produced by a single component whose amplitude $A_{h}$ correspond to Total Harmonic Distortion (THD) level of either 1 or $10 \%$. For this analysis, we vary the harmonic order $h$ from 2 to 50 . For the sake of generality, we also vary the harmonic initial phase within $[0, \pi] \mathrm{rad}$, with an incremental step of $\pi / 10$. In this way, we can evaluate also the effect of phase displacement between the harmonic and the fundamental component.

According to the IEEE Std, TVE and FE limits are equal to $1 \%$ and $5 \mathrm{mHz}$, respectively. The worst-case TVE proves to be independent from the sampling rate, and satisfies the performance requirement in all the considered configurations, not exceeding a maximum value of $0.195 \%$.

Conversely, the worst-case FE varies significantly as function of the distortion level. Given a THD equal to $10 \%$, the frequency error keeps lower than $4 \mathrm{mHz}$. However, limiting the distortion level to $1 \%$, the worst-case FE becomes larger than $80 \mathrm{mHz}$ for a two-cycle window length. In this case, the frequency resolution is too scarce to suitably identify an interfering component with reduced amplitude. As a consequence, the iterative compensation routine might not be successful, and the resulting frequency estimates are gravely distorted. In fact, uncompensated distortion represents one of the main uncertainty sources for any DFT-based synchrophasor estimation algorithm [34], [35].

This effect is noticeable in Fig. 7. The three-cycles configuration is characterized by nearly stable performance TVE and FE around $0.001 \%$ and $0.13 \mathrm{mHz}$, respectively, whereas the two-cycle configuration exhibits a significant accuracy deterioration as the harmonic frequency is lower than $200 \mathrm{~Hz}$.

As regards RFE, the IEEE Std defines a limit of $400 \mathrm{mHz} / \mathrm{s}$. If the THD is set equal to $10 \%$, the worst-case performance is 169 and $137 \mathrm{mHz} / \mathrm{s}$ for two- and three-cycle configuration, respectively. If the THD is set equal to $1 \%$, instead, the HTIpDFT produces a maximum RFE of 216 and $86 \mathrm{mHz} / \mathrm{s}$ for two- and three-cycle configuration, respectively. 
TABLE III

MAXimum TVE AND FE In StATIC TESTS

\begin{tabular}{|c|c|c|c|c|c|c|c|c|c|c|c|c|c|c|c|c|c|c|c|c|}
\hline \multirow[b]{5}{*}{ SNR $[\mathrm{dB}]$} & \multicolumn{10}{|c|}{ TVE [\%] } & \multicolumn{10}{|c|}{$\mathrm{FE}[\mathrm{mHz}]$} \\
\hline & \multirow{2}{*}{\multicolumn{2}{|c|}{ Class }} & \multicolumn{8}{|c|}{ HT-IpDFT } & \multirow{2}{*}{\multicolumn{2}{|c|}{ Class }} & \multicolumn{8}{|c|}{ HT-IpDFT } \\
\hline & & & \multicolumn{4}{|c|}{$F_{s}=10 \mathrm{kHz}$} & \multicolumn{4}{|c|}{$F_{s}=50 \mathrm{kHz}$} & & & \multicolumn{4}{|c|}{$F_{s}=10 \mathrm{kHz}$} & \multicolumn{4}{|c|}{$F_{s}=50 \mathrm{kHz}$} \\
\hline & \multirow[t]{2}{*}{$\mathrm{P}$} & \multirow[t]{2}{*}{ M } & \multicolumn{2}{|c|}{2 cycles } & \multicolumn{2}{|c|}{3 cycles } & \multicolumn{2}{|c|}{2 cycles } & \multicolumn{2}{|c|}{3 cycles } & \multirow[t]{2}{*}{$\mathrm{P}$} & \multirow[t]{2}{*}{ M } & \multicolumn{2}{|c|}{2 cycles } & \multicolumn{2}{|c|}{3 cycles } & \multicolumn{2}{|c|}{2 cycles } & \multicolumn{2}{|c|}{3 cycles } \\
\hline & & & 60 & 80 & 60 & 80 & 60 & 80 & 60 & 80 & & & 60 & 80 & 60 & 80 & 60 & 80 & 60 & 80 \\
\hline Sign Freq & 1 & 1 & 0.02 & 0.003 & 0.02 & 0.002 & 0.01 & 0.001 & 0.01 & 0.001 & 5 & 5 & 4.06 & 0.45 & 2.74 & 0.26 & 1.97 & 0.17 & 1.04 & 0.10 \\
\hline Harm Dist (1\%) & 1 & 1 & 0.16 & 0.154 & 0.11 & 0.090 & 0.19 & 0.187 & 0.01 & 0.001 & 5 & 25 & 86.5 & 83.4 & 2.17 & 0.19 & 84.7 & 83.4 & 1.03 & 0.13 \\
\hline Harm Dist (10\%) & 1 & 1 & 0.10 & 0.090 & 0.11 & 0.090 & 0.01 & 0.007 & 0.01 & 0.001 & 5 & 25 & 3.90 & 0.50 & 1.68 & 0.17 & 2.60 & 0.36 & 1.07 & 0.13 \\
\hline OOBI $(47.5 \mathrm{~Hz})$ & - & 1.3 & 0.11 & 0.091 & 0.11 & 0.087 & 0.03 & 0.025 & 0.01 & 0.008 & - & 10 & 8.23 & 2.90 & 2.55 & 0.39 & 5.78 & 5.28 & 1.25 & 0.95 \\
\hline OOBI $(50 \mathrm{~Hz})$ & - & 1.3 & 0.12 & 0.092 & 0.11 & 0.091 & 0.02 & 0.020 & 0.01 & 0.007 & - & 10 & 7.96 & 1.34 & 3.19 & 0.32 & 6.10 & 4.99 & 1.37 & 0.88 \\
\hline OOBI $(52.5 \mathrm{~Hz})$ & - & 1.3 & 0.19 & 0.165 & 0.19 & 0.163 & 0.05 & 0.022 & 0.02 & 0.009 & - & 10 & 8.24 & 3.34 & 3.90 & 0.39 & 6.08 & 5.13 & 1.41 & 1.03 \\
\hline
\end{tabular}

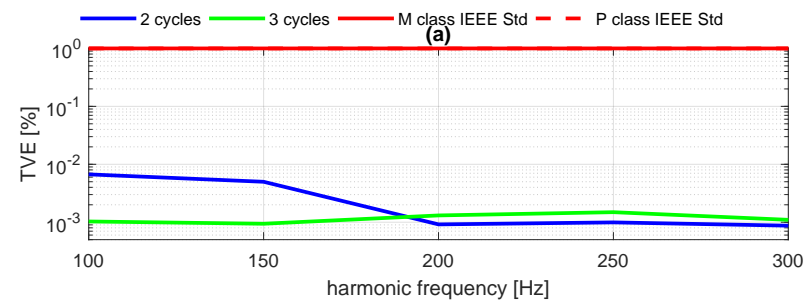

(b)

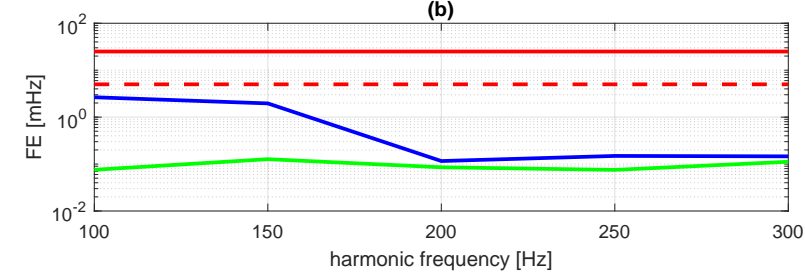

Fig. 7. Harmonic distortion test $(\mathrm{THD}=10 \%)$ : worst-case TVE (a) and FE (b) as function of harmonic component frequency, with 2 (blue) and 3 cycles (green) window. The solid and dashed red lines represent the performance requirements for $\mathrm{M}$ - and P-class, respectively.

The Out-of-band interference test relies on the signal model:

$$
\begin{aligned}
& x(t)=A \cdot \cos \left(2 \pi f t+\varphi_{0}\right)+\eta(t) \\
& \eta(t)=A_{i h} \cdot \cos \left(2 \pi f_{i h} t+\varphi_{i h}\right)
\end{aligned}
$$

where the interfering component has an amplitude $A_{i h}$ corresponding to a Total Inter-Harmonic Distortion (TIHD) of $10 \%$, and its frequency varies within $[10,25]$ or $[75,95]$ Hz. In this regard, we consider an incremental step of $5 \mathrm{~Hz}$, in line with the definition of inter-harmonic group [36], [37].

For this analysis, the fundamental amplitude and initial phase are fixed to $1 \mathrm{pu}$ and $0 \mathrm{rad}$, respectively, whereas the fundamental frequency varies within $[47.5,50,52.5]$ $\mathrm{Hz}$. In this way, it is possible to evaluate how different frequency deviations and phase-shifts between fundamental and interfering components affect the algorithm estimation accuracy.

It is interesting to observe that, for this test, the IEEE Std does not introduce requirements for class $\mathrm{P}$. The compliance to class $\mathrm{M}$, instead, requires TVE and FE not to exceed $1.3 \%$ and $10 \mathrm{mHz}$, respectively. In this regard, the results reported in Table III prove that HT-IpDFT is compliant with the IEEE Std requirements in terms of both TVE and FE in all the considered configurations. As in the previous test, the three-cycle configuration guarantees a significant performance enhancement, due to the fact that a larger window length corresponds to a finer frequency resolution, a reduced short-range interference, and thus a better capability of compensating the spurious tones.
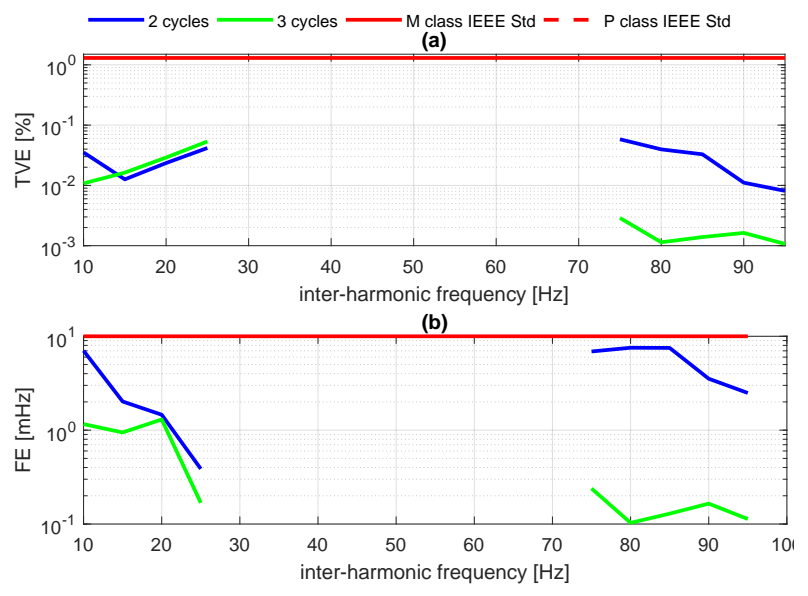

Fig. 8. Out-of-band interference test (TIHD $=10 \%, f=52.5 \mathrm{~Hz})$ : worstcase TVE (a) and FE (b) as function of inter-harmonic component frequency, with 2 (blue) and 3 cycles (green) window. The solid and dashed red lines represent the performance requirements for class $\mathrm{M}$ and $\mathrm{P}$, respectively.

In this context, Fig. 8 presents the worst-case TVEs and FEs as function of the inter-harmonic frequency, obtained with a fundamental frequency of $52.5 \mathrm{~Hz}$.

If $f_{i h}$ is lower than $15 \mathrm{~Hz}$, the frequency resolution of the two-cycle configuration does not allow for discriminating between DC and inter-harmonic contributions. As a consequence, the iterative compensation routine is nonoptimal and produces a TVE and FE equal to $0.02 \%$ and 7 $\mathrm{mHz}$, in the worst-case.

Within $[15,25] \mathrm{Hz}$, the two- and three-cycle configurations provide comparable results: TVE and FE do not exceed $0.05 \%$ and $1 \mathrm{mHz}$, respectively. Finally, if $f_{i h}$ varies within $[75,95] \mathrm{Hz}$, the three-cycle configuration guarantees a performance enhancement by almost one order of magnitude, with a maximum TVE of $0.004 \%$ and a FE lower than 0.2 $\mathrm{mHz}$.

Based on the obtained results, the efficiency of the interference compensation routine cannot be totally guaranteed 
if we consider a window length of just two cycles. In this regard, it should be remembered that the DFT-based spectral analysis has a resolution limit that depends on the adopted window length and weighing function [11]. In the present case, the Hanning weighing function requires a distance of at least two bins to distinguish the interfering tone from the fundamental component. Since in both Harmonic distortion and Out-of-band interference tests this constraint is not satisfied, the compensation routine produces only an approximation of the interfering tone parameters.

The interference compensation routine is characterized by two stop criterion. The first one is related to the residual energy ratio $E_{r}$, whereas the second one sets a maximum number of 100 iterations. In order to prove the effectiveness of the selected criteria, Fig. 9 represents FE and $E_{r}$ as function of the iteration number $\mathrm{Q}$ in two significant cases of the Out-ofBand Interference test, with a two-cycle window.
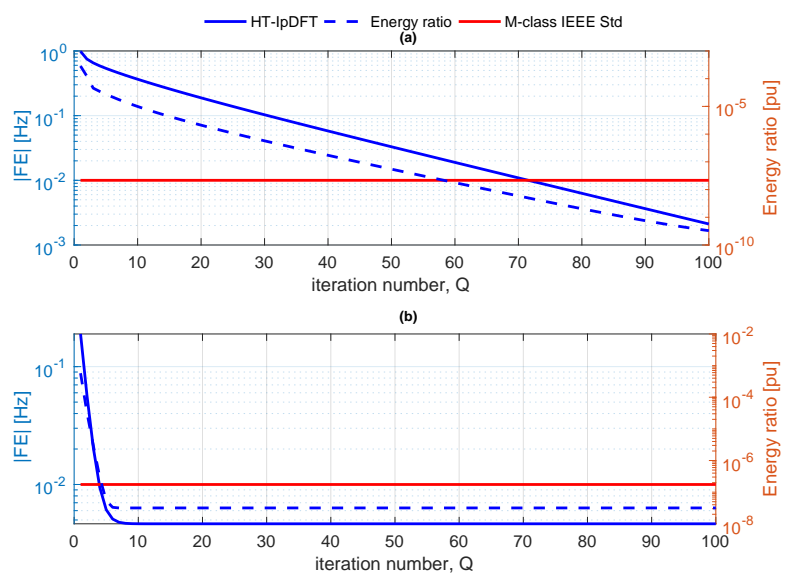

Fig. 9. Frequency Error and Energy ratio as function of the iteration number $\mathrm{Q}$ of the OOBI compensation routine in two-cycle configuration. In (a), we consider $f=52.5 \mathrm{~Hz}$ and $f_{i h}=75 \mathrm{~Hz}$; in (b), $f=50 \mathrm{~Hz}, f_{i h}=10 \mathrm{~Hz}$.

The upper graph (a) considers one of the worst-cases, where $f$ and $f_{i h}$ are set equal to 52.5 and $75 \mathrm{~Hz}$, respectively. As a consequence, the distance between fundamental and inter-harmonic component is less than a single bin on the considered frequency axis. In this challenging scenario, FE and $E_{r}$ decades monotonically but slowly: 72 iterations are necessary to obtain a FE compliant with M-class requirement.

In the lower graph (b), instead, we set the fundamental and inter-harmonic frequency equal to 50 and $10 \mathrm{~Hz}$, respectively. Since the deviation between $f$ and $f_{i h}$ is relevant, it is reasonable to expect that the interference effect is reduced. In fact, FE achieves the compliance with the IEEE Std limit after just four iterations. In a similar way, the residual energy $E_{r}$ rapidly decreases and becomes constant just after 7 iterations.

This example proves how the double stop criterion represents an optimal trade-off between computational efficiency and estimation accuracy. In most the cases, the residual energy determines the end of the compensation routine. Only in the most challenging cases, a high number of iterations is required to achieve the IEEE Std compliance.

b) Dynamic Tests: After assessing the steady-state compliance, the dynamic tests enable us to investigate the
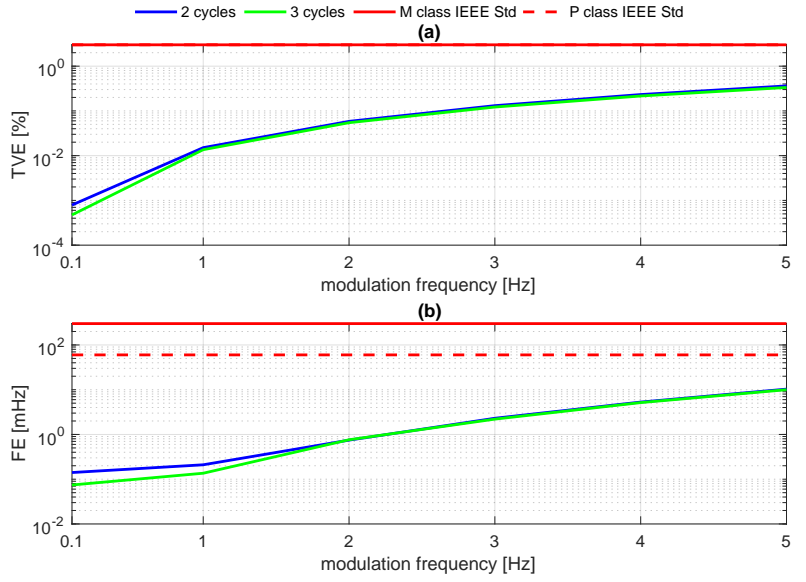

Fig. 10. Amplitude modulation test (depth 10\%): worst-case TVE (a) and FE (b) as function of modulation frequency, with 2 (blue) and 3 cycles (green) window. The solid and dashed red lines represent the performance requirements for $\mathrm{M}$ - and $\mathrm{P}$-class, respectively.

estimation accuracy in the presence of time-varying test conditions. In this context, Table IV reports the results of HTIpDFT.

The Amplitude modulation test relies on the signal model:

$$
\begin{aligned}
x(t) & =A \cdot\left(1+\varepsilon_{A}(t)\right) \cdot \cos \left(2 \pi f t+\varphi_{0}\right) \\
\varepsilon_{A}(t) & =k_{m} \cdot \cos \left(2 \pi f_{m} t\right)
\end{aligned}
$$

where the fundamental amplitude, frequency and initial phase are fixed to their nominal values, whereas $k_{m}$ and $f_{m}$ account for modulation depth and frequency, respectively. In particular, we set $k_{m}$ equal to 0.1 , and we vary $f_{m}$ within $[0.1,5] \mathrm{Hz}$.

In this context, the IEEE Std requires TVE and FE not to exceed $3 \%$ and $60 \mathrm{mHz}$, respectively. Both the constraints are easily met by HT-IpDFT in all the considered configurations, as reported in Table IV. In the worst case, TVE is equal to $0.415 \%$, whereas FE is lower than $13.55 \mathrm{mHz}$.

Fig. 10(a) and (b) represent the worst-case TVEs and FEs as function of the modulation frequency $f_{m}$. It is worth noticing that the estimation accuracy does not depend significantly on the window length. Amplitude, phase and frequency errors are mainly due to the mismatch between the static signal model employed by interpolated DFT and the time-varying trend of the modulated signal. Indeed, comparable results can be obtained also with most recent IpDFT solutions [8].

In terms of RFE, the worst-case performance is equal to 653 and $603 \mathrm{mHz} / \mathrm{s}$ for two- and three-cycle configuration, respectively, whereas the IEEE Std set the limit to $2.3 \mathrm{~Hz} / \mathrm{s}$.

Similar considerations can be made for the Phase modulation test, whose signal model is defined as follows:

$$
\begin{aligned}
x(t) & =A \cdot \cos \left(2 \pi f t+\varphi_{0}+\varepsilon_{\varphi}(t)\right) \\
\varepsilon_{\varphi}(t) & =k_{m} \cdot \cos \left(2 \pi f_{m} t-\pi\right)
\end{aligned}
$$

where the modulation depth $k_{m}$ is equal to $\pi / 18 \mathrm{rad}$, whereas the modulation frequency $f_{m}$ varies between 0.1 and $5 \mathrm{~Hz}$. As in the previous test, the fundamental amplitude, frequency and initial phase are fixed to their nominal values.

With respect to the previous test, the errors have slightly increased. In the worst-case, TVE and FE are equal to $0.925 \%$ 
TABLE IV

MAXIMUM TVE AND FE IN DyNAMIC TESTS

\begin{tabular}{|c|c|c|c|c|c|c|c|c|c|c|c|c|c|c|c|c|c|c|c|c|}
\hline \multirow[b]{5}{*}{ SNR [dB] } & \multicolumn{10}{|c|}{ TVE [\%] } & \multicolumn{10}{|c|}{$\mathrm{FE}[\mathrm{mHz}]$} \\
\hline & \multirow{2}{*}{\multicolumn{2}{|c|}{ Class }} & \multicolumn{8}{|c|}{ HT-IpDFT } & \multirow{2}{*}{\multicolumn{2}{|c|}{ Class }} & \multicolumn{8}{|c|}{ HT-IpDFT } \\
\hline & & & \multicolumn{4}{|c|}{$F_{s}=10 \mathrm{kHz}$} & \multicolumn{4}{|c|}{$F_{s}=50 \mathrm{kHz}$} & & & \multicolumn{4}{|c|}{$F_{s}=10 \mathrm{kHz}$} & \multicolumn{4}{|c|}{$F_{s}=50 \mathrm{kHz}$} \\
\hline & \multirow[t]{2}{*}{$\mathrm{P}$} & \multirow[t]{2}{*}{ M } & $2 c$ & ycles & $3 \mathrm{c}$ & cles & & ycles & & ycles & \multirow[t]{2}{*}{$\mathrm{P}$} & \multirow[t]{2}{*}{ M } & $2 c$ & cles & $3 \mathrm{cy}$ & cles & $2 \mathrm{cs}$ & cles & 3 c) & cles \\
\hline & & & 60 & 80 & 60 & 80 & 60 & 80 & 60 & 80 & & & 60 & 80 & 60 & 80 & 60 & 80 & 60 & 80 \\
\hline Ampl Mod & 3 & 3 & 0.42 & 0.404 & 0.31 & 0.309 & 0.37 & 0.362 & 0.30 & 0.268 & 60 & 300 & 13.2 & 13.0 & 13.5 & 12.9 & 11.1 & 10.3 & 10.7 & 10.0 \\
\hline Ph Mod & 3 & 3 & 0.93 & 0.922 & 0.82 & 0.824 & 0.49 & 0.488 & 0.37 & 0.341 & 60 & 300 & 20.1 & 19.0 & 19.6 & 18.5 & 15.7 & 15.1 & 15.2 & 15.1 \\
\hline Freq Ramp Pos & 1 & 1 & 0.05 & 0.034 & 0.03 & 0.022 & 0.03 & 0.025 & 0.03 & 0.026 & 10 & 10 & 5.44 & 1.60 & 3.05 & 1.52 & 2.87 & 1.32 & 2.01 & 1.27 \\
\hline Freq Ramp Neg & 1 & 1 & 0.05 & 0.035 & 0.05 & 0.039 & 0.03 & 0.022 & 0.03 & 0.021 & 10 & 10 & 5.32 & 2.29 & 3.48 & 2.12 & 3.73 & 1.48 & 2.01 & 1.41 \\
\hline
\end{tabular}
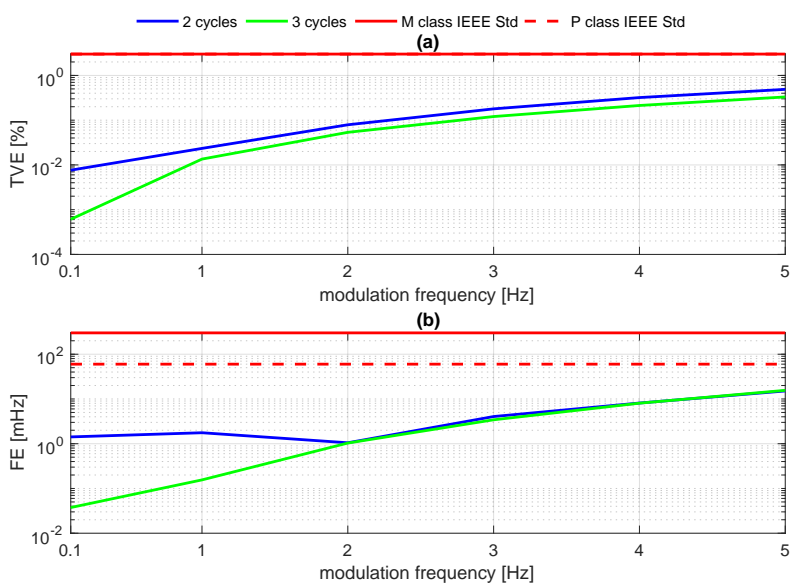

Fig. 11. Phase modulation test (depth $\pi / 18 \mathrm{rad}$ ): worst-case TVE (a) and FE (b) as function of modulation frequency, with 2 (blue) and 3 cycles (green) window. The solid and dashed red lines represent the performance requirements for $\mathrm{M}$ - and $\mathrm{P}$-class, respectively.

and $20.12 \mathrm{mHz}$, respectively. During a phase modulation, both signal frequency and phase are varying. Because of this duality (inherent in the synchrophasor phase angle definition), the possible sources of uncertainties are two. Nevertheless, as shown also in Fig. 11(a) and (b), the proposed algorithm proves to be compliant with the IEEE Std requirements for both class $\mathrm{M}$ and $\mathrm{P}$ in all the considered configurations.

As regards RFE, the worst-case performance is equal to 792 and $694 \mathrm{mHz} / \mathrm{s}$ for two- and three-cycle configuration, respectively, whereas the IEEE Std set the limit to $2.3 \mathrm{~Hz} / \mathrm{s}$.

For the Frequency ramp test, we adopt the signal model:

$$
\begin{aligned}
x(t) & =A \cdot \cos \left(2 \pi f t+\varphi_{0}+\varepsilon_{\varphi}(t)\right. \\
\varepsilon_{\varphi}(t) & =k_{r} \cdot \pi t^{2}
\end{aligned}
$$

where the quadratic phase term corresponds to a linear contribution in frequency. In this context, $k_{r}$ accounts for the rate of change of frequency, as measured in $\mathrm{Hz} / \mathrm{s}$.

For this specific test, the IEEE Std requires the fundamental frequency to vary between 45 and $55 \mathrm{~Hz}$ with a rate of change of $1 \mathrm{~Hz} / \mathrm{s}$, and vice-versa. In terms of accuracy, the worst-case TVE and FE are limited to $1 \%$ and $10 \mathrm{mHz}$, respectively.

The results in Table IV show that HT-IpDFT is compliant with the IEEE Std limits in all the considered configurations. In particular, TVE and FE do not exceed $0.05 \%$ and $5.50 \mathrm{mHz}$. As shown in Fig. 12, the estimation accuracy does not depend on the polarity of the linear ramp: nearly coincident results are obtained for $k_{r}$ equal to +1 or $-1 \mathrm{~Hz} / \mathrm{s}$. As already mentioned for modulation tests, the algorithm performance does not vary significantly when varying the window length: the uncertainty descends from the inconsistency of the IpDFT static signal model, rather than on the frequency resolution.

In this case, the RFE presents a worst-case performance of 189 and $154 \mathrm{mHz} / \mathrm{s}$ for two- and three-cycle configuration, respectively, whereas the IEEE Std set the limit to $200 \mathrm{mHz} / \mathrm{s}$.
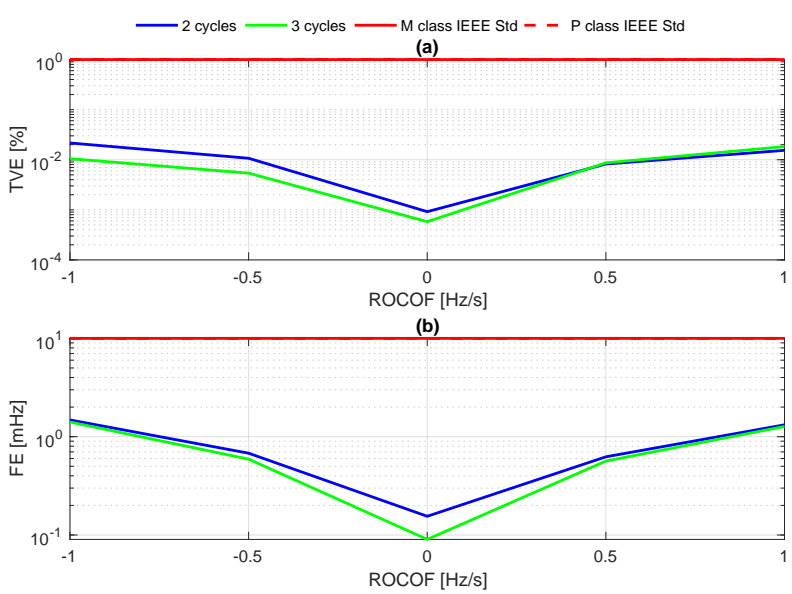

Fig. 12. Frequency ramp test: worst-case TVE (a) and FE (b) as function of the ROCOF, with 2 (blue) and 3 cycles (green) window. The solid and dashed red lines represent the performance requirements for M- and P-class, respectively.

c) Step tests: As final test, we characterize the algorithm performance during an instantaneous step change of signal amplitude or phase. In this way, it is possible to evaluate the algorithm responsiveness to a transient event. In this context, Table V reports the results of HT-IpDFT.

The signal model of the Amplitude step test is defined as:

$$
x(t)=A \cdot\left(1+k_{t} \cdot \chi\left(t-T_{t}\right)\right) \cdot \cos \left(2 \pi f t+\varphi_{0}\right)
$$

where $k_{t}$ and $T_{t}$ account for the step size and occurrence time, respectively, whereas $\chi(\cdot)$ models a piece-wise step function:

$$
\chi(t)= \begin{cases}0 & \text { if } t<0 \\ 1 & \text { if } t \geq 0\end{cases}
$$

For this analysis, we set $k_{t}$ and $T_{t}$ to 0.1 and $1.5 \mathrm{~s}$, respectively.

In this case, the IEEE Std requirements regard the algorithm response time, defined as the time to transition between two 
TABLE V

Maximum Response Time [ms] for TVE and FE in Step Tests

\begin{tabular}{|c|c|c|c|c|c|c|c|c|c|c|c|c|c|c|c|c|c|c|c|c|}
\hline \multirow[b]{5}{*}{ SNR [dB] } & \multicolumn{10}{|c|}{ TVE Resp Time [ms] } & \multicolumn{10}{|c|}{ FE Resp Time [ms] } \\
\hline & \multirow{2}{*}{\multicolumn{2}{|c|}{ Class }} & \multicolumn{8}{|c|}{ HT-IpDFT } & \multirow{2}{*}{\multicolumn{2}{|c|}{ Class }} & \multicolumn{8}{|c|}{ HT-IpDFT } \\
\hline & & & \multicolumn{4}{|c|}{$F_{s}=10 \mathrm{kHz}$} & \multicolumn{4}{|c|}{$F_{s}=50 \mathrm{kHz}$} & \multirow{3}{*}{$\mathrm{P}$} & & \multicolumn{4}{|c|}{$F_{s}=10 \mathrm{kHz}$} & \multicolumn{4}{|c|}{$F_{s}=50 \mathrm{kHz}$} \\
\hline & \multirow[t]{2}{*}{$\mathrm{P}$} & \multirow[t]{2}{*}{$\mathrm{M}$} & $2 \mathrm{cy}$ & cles & $3 c$ & cles & $2 \mathrm{c}$ & cles & $3 \mathrm{cy}$ & cles & & \multirow[t]{2}{*}{ M } & $2 \mathrm{cl}$ & cles & $3 c$ & cles & $2 \mathrm{cl}$ & cles & $3 \mathrm{cl}$ & cles \\
\hline & & & 60 & 80 & 60 & 80 & 60 & 80 & 60 & 80 & & & 60 & 80 & 60 & 80 & 60 & 80 & 60 & 80 \\
\hline Ampl step & 40 & 140 & 22.9 & 22.9 & 32.8 & 32.5 & 23.5 & 23.5 & 35.8 & 35.8 & 90 & 280 & 64.4 & 63 & 57.8 & 57.1 & 62 & 61.2 & 55.8 & 55.8 \\
\hline $\mathrm{Ph}$ step & 40 & 140 & 26.5 & 26.4 & 82.5 & 82 & 36.2 & 36.1 & 71.1 & 71.1 & 90 & 280 & 84.9 & 84.5 & 143.4 & 140.7 & 94.4 & 94 & 123.6 & 120.7 \\
\hline
\end{tabular}

steady-state measurements before and after the step change. It is determined as the difference between the instants when the measurement accuracy exits and reenters within a given limit (i.e. $\mathrm{TVE} \leq 1 \%, \mathrm{FE} \leq 5 \mathrm{mHz}$ ). This requirement is particularly stringent for class $\mathrm{P}$ : the response time should not exceed 40 and $90 \mathrm{~ms}$ for TVE and FE, respectively.

This analysis cannot be performed with the canonical reporting rate of $50 \mathrm{fps}$. It is necessary to compute the interleaved time-domain trend of TVE and FE, as indicated by the IEEE Std specifications. To this end, we set $F_{r}$ equal to $F_{s}$ producing a new estimate in correspondence with each acquired sample.

In Fig. 13, the solid blue and green lines represent TVE and FE as function of time. The error trends obtained with two and three cycles have been aligned with respect to $T_{\text {start }}$, i.e. the time instant where the performance index exceeds the corresponding threshold (dashed black line). The dashed lines indicate the step occurrence time $T_{t}$ in the two configurations.

Both TVE and FE are characterized by a response time lower than the class $\mathrm{P}$ limit (dashed red line). As reported in Table $\mathrm{V}$, the worst-case response time is equal to 35.8 and $64.4 \mathrm{~ms}$ for TVE and FE, respectively.

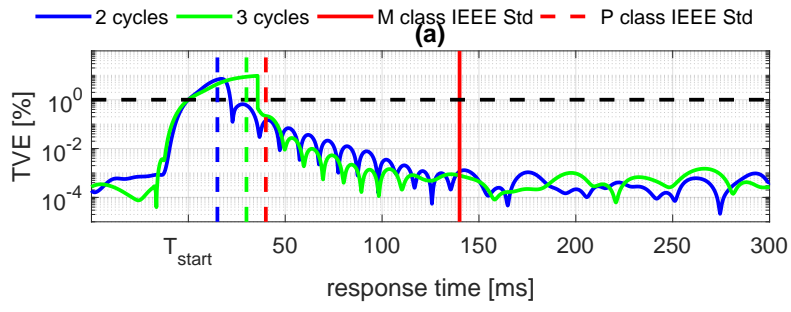

(b)

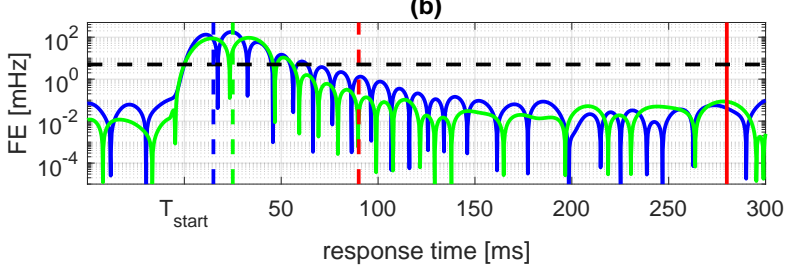

Fig. 13. Amplitude step test $(+0.1)$ : interleaved analysis of TVE (a) and FE (b), with 2 (blue) and 3 cycles (green) window. The solid and dashed red lines represent the performance requirements for class $\mathrm{M}$ and $\mathrm{P}$, respectively, whereas the dashed black line represents the corresponding accuracy limits.

In line with (15), the Phase step signal model is:

$$
x(t)=A \cdot \cos \left(2 \pi f t+\varphi_{0}+k_{t} \cdot \chi\left(t-T_{t}\right)\right)
$$

where the step size $k_{t}$ corresponds to $\pi / 18 \mathrm{rad}$, and the step occurrence time $T_{t}$ is set equal to $1.5 \mathrm{~s}$.
In Table $\mathrm{V}$, we notice a degradation of the algorithm responsiveness as the window length is increased. None of the three-cycle configurations is compliant with the requirements of $\mathrm{P}$ class, in terms of neither TVE nor FE. Conversely, the two-cycle configurations guarantee a satisfactory estimation accuracy, with the only exception of sampling frequency equal to $50 \mathrm{kHz}$, where the worst-case FE response time exceeds the limit by less than $5 \mathrm{~ms}$. On the other hand, it is important to notice that all the considered configurations are compliant with the requirements of class $M$.
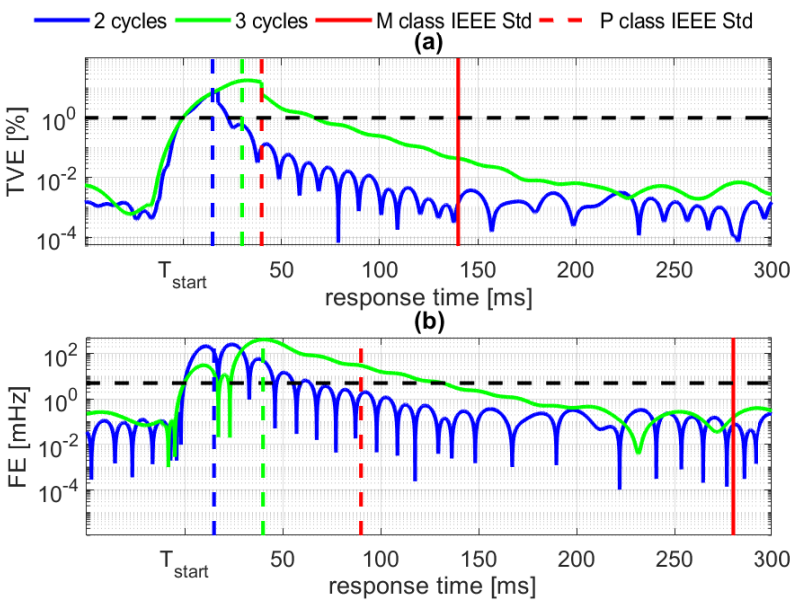

Fig. 14. Phase step test $(+\pi / 18 \mathrm{rad})$ : interleaved analysis of TVE (a) and FE (b), with 2 (blue) and 3 cycles (green) window. The solid and dashed red lines represent the performance requirements for class $\mathrm{M}$ and $\mathrm{P}$, respectively, whereas the dashed black line represents the corresponding accuracy limits.

With respect to the previous test, the reason for such a performance deterioration can be found in the filter frequency response. Given the limited order, the Fast HT Filter is characterized by a step response with a reduced settling time, but a non-negligible ringing effect: the cosine argument exhibits a damped oscillation that can be interpreted as either a phase or frequency modulation. In fact, the synchrophasor estimation algorithm is unable to discriminate between these contributions, due to the ambivalent definition of synchrophasor phase angle depending on both fundamental frequency and phase [40], [41]. Therefore, the same damped oscillation is noticeable in TVE and FE trends in Fig. 14, whereas in the Amplitude step test, this trend was restricted to only the TVE.

It should be noticed that an analysis based only on TVE and $\mathrm{FE}$ in the presence of transient events might lack of consistency. The IEEE Std relies on a synchrophasor 
model that assumes the signal DFT representation to consist of few narrow-band components: a term associated to the fundamental component, plus a restricted set of harmonic or inter-harmonic contributions. In practical conditions, though, this finite spectrum assumption might not be able to suitably represent quasi-instantaneous variations of signal parameters and the definition of TVE might not be the most appropriate metric for assessing the accuracy of synchrophasor estimation algorithms [42].

For this reason, we perform also a time-domain analysis, intended to quantify the discrepancy between the HT-IpDFT estimates and the acquired waveform. At each reporting period, we employ the estimated values $A^{*}, f^{*}$ and $\varphi_{0}^{*}$ to recover the time-domain trend of the fundamental component as:

$$
x^{*}[n]=A^{*} \cdot \cos \left(2 \pi f^{*} n T_{s}+\varphi_{0}^{*}\right), \quad n=\left[1, \ldots N_{m}\right]
$$

Then, we compute the normalized Root Mean Squared Error (nRMSE) between the recovered and original waveform:

$$
n R M S E=\sqrt{\sum_{n=1}^{N_{m}} \frac{\left(x^{*}[n]-x[n]\right)^{2}}{N_{m}}}
$$

It should be noticed that nRMSE is not intended as a replacement of the canonical TVE, but as a numerical index of the estimation reliability [42]. Therefore, at each reporting period, we are able to provide not only the estimated amplitude, frequency, and initial phase, but also the corresponding nRMSE, that quantifies the discrepancy between what the PMU is acquiring and what the PMU is estimating. The recent literature proposes a similar metric, called Goodness of Fit, that depends on the residual energy, as well as on the number of degrees of freedom of the considered signal model [43].

In this context, Fig. 15 presents the nRMSEs as measured during both Amplitude and Phase step tests as function of time. Before and after the step change, the nRMSE accounts only for the measurement noise, with an average value of about $10^{-4} \mathrm{pu}$ that corresponds exactly to the expected SNR of 80 $\mathrm{dB}$. As the acquired waveform includes also the step change, the nRMSE rapidly increases up to $10^{-2} \mathrm{pu}$. In fact, during the transient event the signal energy is conveyed through the entire spectrum and the synchrophasor model does not represent a reliable approximation of the observed phenomenon.

Based on nRMSE, it is also possible to investigate the SE algorithm responsiveness, following a procedure similar to the one employed for the response time computation. In this case, we adopt as performance limit a threshold level computed as three times the expected noise level, i.e. $3 \cdot 10^{-4} \mathrm{pu}$. Then, we compute the time difference between the threshold crossings.

In this context, it is interesting to compare these time interval duration with TVE and FE response times. However, it should be noticed that the nRMSE metric account for the cumulative estimation uncertainty due to amplitude, frequency, and initial phase error. Moreover, the adopted threshold represents a more challenging limit with respect to a maximum TVE or FE equal to $1 \%$ and $5 \mathrm{mHz}$, respectively. By setting the threshold equal to the expected noise level, we are measuring
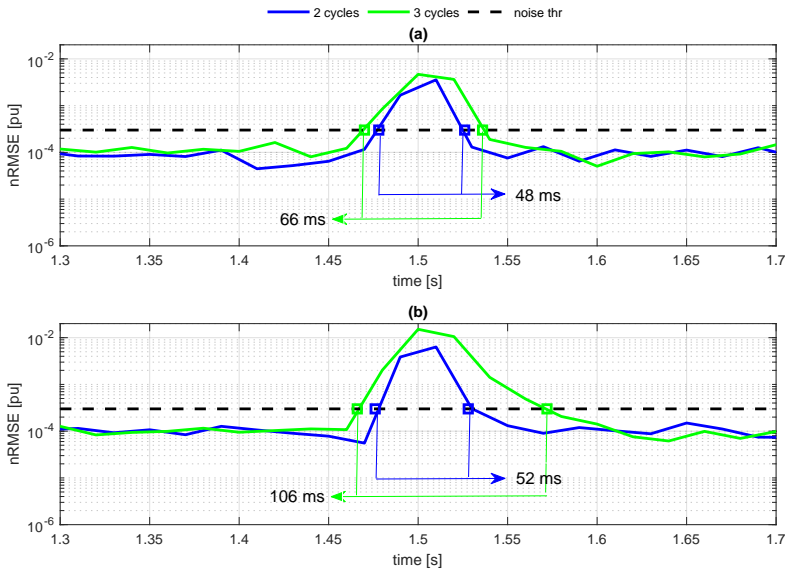

Fig. 15. Normalized root-mean-squared error during amplitude (a) and phase (b) step test for window length equal to 2 (blue) and 3 cycles (green). The dashed black line represents the reliability threshold, computed as three times the expected noise level.

the time interval necessary to return to an estimation accuracy typical of steady-state test conditions.

With respect to TVE and FE response times, the nRMSE analysis confirms the advantage of the two-cycle configuration, characterized by a prompter adjustment to the transient event, as well as the performance deterioration in the Phase step test. In this regard, it is worth observing that the nRMSEbased time interval is strictly lower than the FE response time. This result demonstrates that the damped oscillations observed in Fig. 14 are due to frequency and phase errors that are partially compensating each other. For this reason, a time-domain analysis that does not suffer from this duality inherent in the synchrophasor model definition might be a more effective solution to study the reliability of the algorithm estimates in the presence of transient events.

d) Performance summary: As an aggregated result summary, Table VI reports the worst-case TVEs, FEs, and RFEs provided by the HT-IpDFT in all the considered configurations for each test condition defined by the IEEE Std, including all considered noise levels and window lengths.

In this regard, it is worth noticing that TVE does not exceed $1 \%$ and we keep FE and RFE within $90 \mathrm{mHz}$ and 800 $\mathrm{mHz} / \mathrm{s}$, respectively, even considering a DFT-based frequency resolution of just $25 \mathrm{~Hz}$.

\section{COMPARISON With IPDFT State OF THE ART}

In this section, we compare the estimation accuracy of the proposed HT-IpDFT with other two IpDFT-based synchrophasor estimation algorithms. To this end, in the present analysis we consider the Enhanced IpDFT, briefly e-IpDFT [12], and the Iterative IpDFT, briefly i-IpDFT [8]. On one side, e-IpDFT relies on a two-point IpDFT and iteratively compensates the long-range spectral leakage due to the negative image of the fundamental component. On the other side, i-IpDFT employs a three-point IpDFT and iteratively estimates and compensates the spectral leakage effects due to not only the fundamental component, but also generic interfering tones. This comparison enables us to 
TABLE VI

Worst-CASE Performance of HT-IPDFT in IEEE Std Tests

\begin{tabular}{c|ccc}
\hline Test & TVE [\%] & FE [mHz] & RFE [mHz/s] \\
\hline Sign Freq & 0.02 & 4.06 & 3.6 \\
\hline Harm Dist (1\%) & 0.19 & 86.5 & 216 \\
Harm Dist (10\%) & 0.11 & 3.90 & 169 \\
\hline OOBI & 0.19 & 8.24 & 264 \\
\hline Ampl Mod & 0.42 & 13.5 & 653 \\
Ph Mod & 0.93 & 20.1 & 792 \\
\hline Freq Ramp & 0.05 & 5.44 & 189 \\
\hline \hline \multicolumn{2}{c}{ Response Time } & TVE [ms] & FE [ms] \\
\hline \multicolumn{2}{c}{ Ampl Step } & 35.8 & 64.4 \\
Ph Step & 82.5 & 143.4 \\
\hline
\end{tabular}

determine which algorithm is more successful in mitigating the spectral leakage effects, even when significant spurious contributions affect the test signal.

Since the rejection of interferences is one of the major issues for IpDFT-based algorithms, we focus on the estimation accuracy provided by HT-IpDFT, e-IpDFT and i-IpDFT in both Harmonic distortion and Out-of-band interference test. For this analysis, the amplitude of the interference tone is set equal to $0.1 \mathrm{pu}$, corresponding to a THD and TIHD of $10 \%$. In this context, Fig. 16 presents the maximum FE as function of the interfering tone frequency. The sampling frequency and SNR are set equal to $50 \mathrm{kHz}$ and $80 \mathrm{~dB}$, respectively.

More precisely, Fig. 16(a) refers to a window length of two cycles, i.e. $40 \mathrm{~ms}$. As the interference frequency is lower than $25 \mathrm{~Hz}$, only the HT-IpDFT solution proves to be compliant with the IEEE Std requirements for M-class. The motivation of this significant performance enhancement can be found in the interference compensation routine: not implemented in e-IpDFT, and ineffective in i-IpDFT. In this regard, it is worth noticing that the proximity with the negative frequency components makes the compensation process more difficult.

Similar results are obtained for interference frequencies larger than $75 \mathrm{~Hz}$. In this context, e-IpDFT does not comply with the IEEE Std requirements, whereas i-IpDFT exceeds the performance limit only for $f_{i h}=[75,80] \mathrm{Hz}$. Conversely, HT-IpDFT guarantees the FE to be compliant with the IEEE Std requirements for the entire considered frequency range.

In Fig. 16(b) we consider a three-cycle window length. In this case, HT-IpDFT and i-IpDFT provide nearly coincident results. Only in the frequency range $[10,25] \mathrm{Hz}$, the Hilbert formulation produces a slight performance enhancement, thanks to the mitigation of the long-range spectral leakage effects from the negative frequency components. On the other hand, e-IpDFT cannot compensate the out-of-band injections, and complies only with the Harmonic distortion test requirements.

\section{CONCLUSION}

In this paper, we present a novel synchrophasor estimation algorithm based on the joint application of the HT and IpDFT. By considering the analytic signal representation,
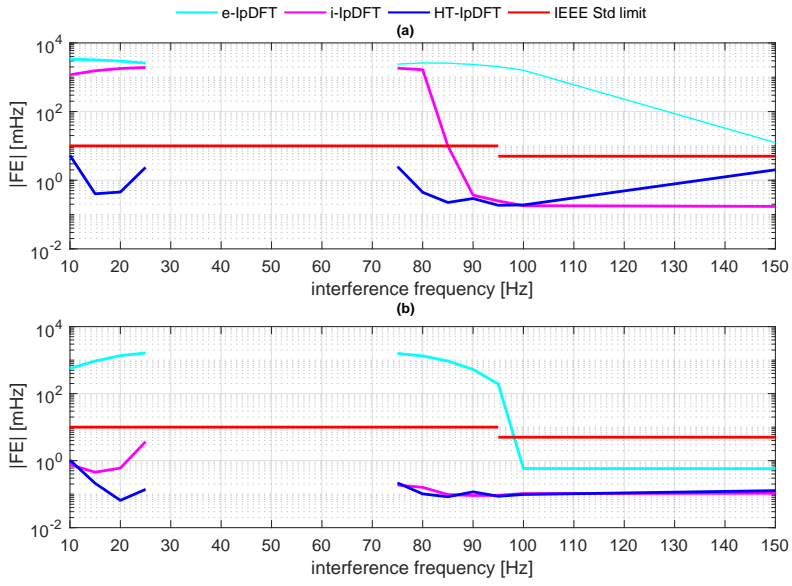

Fig. 16. Estimation accuracy comparison between e-IpDFT (cyan), i-IpDFT (magenta) and HT-IpDFT (blue) with two different window lengths, i.e. 40 $\mathrm{ms}$ in (a), and $60 \mathrm{~ms}$ in (b). Maximum FEs as function of the interference frequency are compared to the IEEE Std most stringent limits (red): $10 \mathrm{mHz}$ for Out-of-band interference test, and $5 \mathrm{mHz}$ for Harmonic distortion test.

we are able to mitigate the long-range spectral leakage effect caused by the negative frequency components and thus reduce significantly the window length up to $40 \mathrm{~ms}$. In this way, we aim at providing high-accuracy estimates of synchrophasor, frequency, and ROCOF associated to the fundamental component, as well as minimizing the measurement reporting latency.

First, we describe the theoretical principles and the implementation details of the proposed HT-IpDFT algorithm, providing a preliminary analysis of its computational requirements and discussing the most suitable setting of the algorithm parameters. Then, we characterize its metrological performance in terms of estimation accuracy and response time in all the test conditions indicated by the IEEE Std. In the context of step tests, we also discuss the validity of the canonical synchrophasor representation in the presence of transient variations, and we propose the adoption of an alternative time domain metric to assess the estimation reliability. Finally, a comparison with other IpDFT-based algorithms proves that the HT-IpDFT is more accurate and robust in the presence of out-of-band and low-order harmonic interferences.

Based on the obtained results, the employment of Hilbert Transform represents a promising solution for synchrophasor estimation, as it allows for a relaxation of the reporting latency constraints and a restraint of the computational complexity. An IpDFT-based approach guarantees the compliance with all the IEEE Std requirements for both $\mathrm{M}$ and $\mathrm{P}$ class, with the only exception of Harmonic distortion (1\%) and Phase step tests. As a consequence, the future steps of the research activity will involve: a more effective routine for the detection and compensation of small amplitude interfering tones, and a more stable though fast response of the filter cascade employed to compute the analytic signal.

\section{REFERENCES}

[1] J. De La Ree, V. Centeno, J. S. Thorp and A. G. Phadke, "Synchronized Phasor Measurement Applications in Power Systems," in 
IEEE Transactions on Smart Grid, vol. 1, no. 1, pp. 20-27, June 2010.

[2] J. Liu, J. Tang, F. Ponci, A. Monti, C. Muscas and P. A. Pegoraro, ”TradeOffs in PMU Deployment for State Estimation in Active Distribution Grids," in IEEE Transactions on Smart Grid, vol. 3, no. 2, pp. 915-924, June 2012.

[3] M. Chakir, I. Kamwa and H. Le Huy, "Extended C37.118.1 PMU Algorithms for Joint Tracking of Fundamental and Harmonic Phasors in Stressed Power Systems and Microgrids," in IEEE Transactions on Power Delivery, vol. 29, no. 3, pp. 1465-1480, June 2014.

[4] P. Castello, J. Liu, C. Muscas, P. A. Pegoraro, F. Ponci and A. Monti, "A Fast and Accurate PMU Algorithm for P $+M$ Class Measurement of Synchrophasor and Frequency," in IEEE Transactions on Instrumentation and Measurement, vol. 63, no. 12, pp. 2837-2845, Dec. 2014.

[5] "IEEE Standard for Synchrophasor Measurements for Power Systems," in IEEE Std C37.118.1-2011 (Revision of IEEE Std C37.118-2005), vol., no., pp.1-61, Dec. 282011

[6] "IEEE Standard for Synchrophasor Measurements for Power Systems Amendment 1: Modification of Selected Performance Requirements," in IEEE Std C37.118.1a-2014 (Amendment to IEEE Std C37.118.1-2011), vol., no., pp.1-25, April 302014.

[7] P. Castello, M. Lixia, C. Muscas and P. A. Pegoraro, "Impact of the Model on the Accuracy of Synchrophasor Measurement," in IEEE Transactions on Instrumentation and Measurement, vol. 61, no. 8, pp. 2179-2188, Aug. 2012.

[8] A. Derviškadić, P. Romano and M. Paolone, "Iterative-Interpolated DFT for Synchrophasor Estimation: A Single Algorithm for P- and MClass Compliant PMUs," in IEEE Transactions on Instrumentation and Measurement, vol. 67, no. 3, pp. 547-558, March 2018

[9] J. W. Stahlhut, T. J. Browne, G. T. Heydt and V. Vittal, "Latency Viewed as a Stochastic Process and its Impact on Wide Area Power System Control Signals," in IEEE Transactions on Power Systems, vol. 23, no. 1, pp. 84-91, Feb. 2008

[10] G. Barchi, D. Macii, and D. Petri, "Synchrophasor Estimators Accuracy: A Comparative Analysis," IEEE Transactions on Instrumentation and Measurement, vol. 62, no. 5, pp. 963-973, May 2013.

[11] F. J. Harris, "On the use of windows for harmonic analysis with the discrete Fourier transform," in Proceedings of the IEEE, vol. 66, no. 1, pp. 51-83, Jan. 1978.

[12] P. Romano and M. Paolone, "Enhanced Interpolated-DFT for Synchrophasor Estimation in FPGAs: Theory, Implementation, and Validation of a PMU Prototype," IEEE Transactions on Instrumentation and Measurement, vol. 63, no. 12, pp. 2824-2836, Dec 2014.

[13] D. Belega and D. Petri, "Accuracy Analysis of the Multicycle Synchrophasor Estimator Provided by the Interpolated DFT Algorithm," in IEEE Transactions on Instrumentation and Measurement, vol. 62, no. 5, pp. 942-953, May 2013.

[14] M. A. Platas-Garza, J. Platas-Garza, and J. A. de la O Serna, "Dynamic Phasor and Frequency Estimates through Maximally Flat Differentiators," in IEEE Transactions on Instrumentation and Measurement, vol. 59, no. 7, pp. 1803-1811, July 2010.

[15] D. Belega, D. Fontanelli and D. Petri, "Dynamic Phasor and Frequency Measurements by an Improved Taylor Weighted Least Squares Algorithm," in IEEE Transactions on Instrumentation and Measurement, vol. 64, no. 8, pp. 2165-2178, Aug. 2015.

[16] M. Bertocco, G. Frigo, C. Narduzzi, C. Muscas, and P. Pegoraro, "Compressive Sensing of a Taylor-Fourier Multifrequency Model for Synchrophasor Estimation," Instrumentation and Measurement, IEEE Transactions on, vol. 64, no. 12, pp. 3274-3283, Dec 2015.

[17] M. Bertocco, G. Frigo, C. Narduzzi, and F. Tramarin, "Resolution Enhancement by Compressive Sensing in Power Quality and Phasor Measurement," IEEE Transactions on Instrumentation and Measurement, vol. 63 , no. 10 , pp. $2358-2367$, Oct 2014.

[18] W. Meng, X. Wang, Z. Wang and I. Kamwa, "Impact of Causality on Performance of Phasor Measurement Unit Algorithms," in IEEE Transactions on Power Systems, vol. 33, no. 2, pp. 1555-1565, March 2018.

[19] V. K. Jain, W. L. Collins and D. C. Davis, "High-Accuracy Analog Measurements via Interpolated FFT," in IEEE Transactions on Instrumentation and Measurement, vol. 28, no. 2, pp. 113-122, June 1979.

[20] Daniel Belega, Dario Petri, and Dominique Dallet, "Frequency estimation of a sinusoidal signal via a three-point interpolated DFT method with high image component interference rejection capability," in Digital Signal Processing, vol. 24, pp. 162-169, Jan. 2014.

[21] C. Qian and M. Kezunovic, "A Power Waveform Classification Method for Adaptive Synchrophasor Estimation," in IEEE Transactions on Instrumentation and Measurement, vol. 67, no. 7, pp. 1646-1658, July 2018.
[22] M. Sahani and P. K. Dash, "Automatic Power Quality Events Recognition based on Hilbert Huang Transform and Extreme Learning Machine," in IEEE Transactions on Industrial Informatics (Early Access), Feb. 2018.

[23] I. Urbina-Salas, J. R. Razo-Hernandez, D. Granados-Lieberman, M. Valtierra-Rodriguez and J. E. Torres-Fernandez, "Instantaneous Power Quality Indices Based on Single-Sideband Modulation and Wavelet Packet-Hilbert Transform," in IEEE Transactions on Instrumentation and Measurement, vol. 66, no. 5, pp. 1021-1031, May 2017.

[24] S. Affijulla and P. Tripathy, "Development of Phasor Estimation Algorithm for P-Class PMU Suitable in Protection Applications," in IEEE Transactions on Smart Grid, vol. 9, no. 2, pp. 1250-1260, March 2018

[25] P. Henrici, "Applied and Computational Complex Analysis, Volume 1: Power Series Integration Conformal Mapping Location of Zero," John Wiley \& Sons, 1988.

[26] M. Alavi-Sereshki and J. Prabhakar, "A Tabulation of Hilbert Transforms for Electrical Engineers," in IEEE Transactions on Communications, vol. 20, no. 6, pp. 1194-1198, Dec 1972.

[27] R. N. Bracewell, "The Fourier Transform and Its Applications," McGraw-Hill, 1963.

[28] V. Cizek, "Discrete Hilbert transform," in IEEE Transactions on Audio and Electroacoustics, vol. 18, no. 4, pp. 340-343, December 1970.

[29] M. Vetterli, J. Kovacevic, V. K. Goyal, "Foundations of Signal Processing," Cambridge University Press, 2014.

[30] D. Petri, D. Fontanelli and D. Macii, "A Frequency-Domain Algorithm for Dynamic Synchrophasor and Frequency Estimation," in IEEE Transactions on Instrumentation and Measurement, vol. 63, no. 10, pp. 2330-2340, Oct. 2014.

[31] I. Kollar, R. Pintelon and J. Schoukens, "Optimal FIR and IIR Hilbert transformer design via $L S$ and minimax fitting," in IEEE Transactions on Instrumentation and Measurement, vol. 39, no. 6, pp. 847-852, Dec. 1990.

[32] M. Sabri and W. Steenaart, "Discrete Hilbert transform filtering," in IEEE Transactions on Acoustics, Speech, and Signal Processing, vol. 25, no. 5, pp. 452-454, October 1977.

[33] T. Grandke, "Interpolation Algorithms for Discrete Fourier Transforms of Weighted Signals," in IEEE Transactions on Instrumentation and Measurement, vol. 32, no. 2, pp. 350-355, June 1983.

[34] D. Agrez, "Weighted Multipoint Interpolated DFT to Improve Amplitude Estimation of Multifrequency Signal," in IEEE Transactions on Instrumentation and Measurement, vol. 51, no. 2, pp. 287-292, Apr 2002.

[35] C. Narduzzi, M. Bertocco, G. Frigo and G. Giorgi, "FastTFM-Multifrequency Phasor Measurement for Distribution Networks," in IEEE Transactions on Instrumentation and Measurement, vol. 67, no. 8, pp. 1825-1835, Aug. 2018.

[36] IEEE Interharmonic Task Force, Cigré 36.05/CIRED 2 CC02 Voltage Quality Working Group. Interharmonics in Power Systems. [Online]. Available: http://grouper.ieee.org/groups/harmonic/iharm/ihfinal.pdf

[37] A. Testa, D. Gallo and R. Langella, "On the Processing of harmonics and interharmonics: using Hanning window in standard framework," in IEEE Transactions on Power Delivery, vol. 19, no. 1, pp. 28-34, Jan. 2004.

[38] W. Premerlani, B. Kasztenny, and M. Adamiak, "Development and Implementation of a Synchrophasor Estimator Capable of Measurements under Dynamic Conditions," IEEE Transactions on Power Delivery, vol. 23, no. 1, pp. 109-123, Jan 2008.

[39] G. Barchi, D. Macii, D. Belega, and D. Petri, "Performance of Synchrophasor Estimators in Transient Conditions: A Comparative Analysis," IEEE Transactions on Instrumentation and Measurement, vol. 62, no. 9, pp. 2410-2418, Sept 2013.

[40] A. J. Roscoe, A. Dyśko, B. Marshall, M. Lee, H. Kirkham and G. Rietveld, "The Case for Redefinition of Frequency and ROCOF to Account for AC Power System Phase Steps," 2017 IEEE International Workshop on Applied Measurements for Power Systems (AMPS), Liverpool, 2017, pp. 1-6.

[41] A. Riepnieks, H. Kirkham, A. J. Faris and M. Engels, "Phase Jumps in PMU Signal Generators," 2017 IEEE Power \& Energy Society General Meeting, Chicago, IL, 2017, pp. 1-5.

[42] G. Frigo, D. Colangelo, A. A. Derviškadić, M. Pignati, C. Narduzzi and M. Paolone, "Definition of Accurate Reference Synchrophasors for Static and Dynamic Characterization of PMUs," in IEEE Transactions on Instrumentation and Measurement, vol. 66, no. 9, pp. 2233-2246, Sept. 2017. 
[43] A. Riepnieks and H. Kirkham, "An Introduction to Goodness of Fit for PMU Parameter Estimation," in IEEE Transactions on Power Delivery, vol. 32, no. 5, pp. 2238-2245, Oct. 2017. 